\title{
SIRPo sequesters SHP-2 to promote IL-4/13 signaling and alternative activation of macrophages
}

\author{
Lei Shi ${ }^{1}$, Koby Kidder ${ }^{1}$, Zhen Bian ${ }^{1}$, Samantha Kuon Ting Chiang ${ }^{1}$, Corbett Ouellette ${ }^{1}$, Yuan Liu ${ }^{1,2 *}$
}

${ }^{1}$ Program of Immunology \& Molecular Cellular Biology, Department of Biology, and ${ }^{2}$ Center of Diagnostics and Therapeutics, Georgia State University, Atlanta, GA 30302

One-sentence summary: By restraining SHP-2, SIRP $\alpha$ disinhibits IL-4/13 signaling to promote macrophage alternative activation and ensure proper resolution of inflammation.

*Corresponding author:

Yuan Liu, MD, PhD

Professor

Center for Diagnostics \& Therapeutics

\& Department of Biology

Georgia State University

Atlanta, GA 30303

Tel.: 404-413-5407

Email: yliu@gsu.edu 


\begin{abstract}
The Th2 cytokines IL-4 and IL-13 through activation of their shared receptor IL-4R $\alpha$ direct macrophage alternative activation to promote immunosuppression and wound healing. However, the mechanisms that control macrophage responses to IL-4/13 are not fully understood. Apart from driving JAK-STAT and PI3K-Akt pathways to polarize macrophages toward the alternative phenotype, the activated IL-4/13 receptors recruit negative regulators SHP-1 and SHP-2, which dephosphorylate IL-4R $\alpha$ and decrease its signaling. Here we report that SIRP $\alpha$ spatially restricts SHP-2 and, by such, promotes IL-4/13 signaling and macrophage alternative activation. SIRP $\alpha$ executes this regulation via its cytoplasmic ITIMs/ITSMs that undergo phosphorylation by IL4/13-induced, Src kinase-activated Bruton's tyrosine kinase (Btk), resulting in recruitment of SHP-2 and preclusion of SHP-2 from binding to and inhibiting IL-4/13 receptors. Despite that this regulation occurs independent of CD47, extracellular CD47 ligation of SIRP $\alpha$ facilitates its cytoplasmic phosphorylation and SHP2 sequestration, leading to stronger IL-4/13 signaling and enhanced macrophage expression of IL-10, TGF $\beta$, CD206, arginase-1, etc. Conversely, deficiency of SIRP $\alpha$ allows SHP-2 to freely bind to $\gamma \mathrm{C}$ or IL-13R $\alpha 1$ and through which dephosphorylate IL-4R $\alpha$, dampening its signaling. Consistent with these findings, impaired wound healing in Sirpo ${ }^{-/-}$mice under experimental colitis correlated with a deficit of immunosuppressive macrophages in the colon, a condition that was corrected by transfusion of ex vivo-produced SIRP ${ }^{\text {high }}$ alternatively activated macrophages.
\end{abstract}




\section{Introduction}

Alternative activation (M2/M2a) of macrophages by the Th2 cytokines IL-4 and/or IL-13 plays an important role in macrophage-driven clearance of parasitic infection, resolution of inflammation and wound healing, as well as dysregulated pathological conditions including allergy, excessive fibrosis and immunosuppressive milieus conducive to tumor growth and metastasis $(1,2)$. To drive the typical M2a phenotype, IL-4 and IL-13 bind to their cognate receptor chain, IL-4R $\alpha$ and IL-13R $\alpha 1$, respectively, and induce receptor and co-receptor dimerization forming IL-4R $\alpha / \gamma \mathrm{C}$ and/or IL-4R $\alpha / \mathrm{IL}-13 \mathrm{R} \alpha 1$ heterodimers, which enable activation of their associated Janus kinases (JAK1/3, Tyk2) to phosphorylate the receptor cytoplasmic domains for the docking of downstream signaling molecules including STAT6, Gab2 and insulin receptor substrate (IRS2) (3-5). After these signaling molecules dock to cytokine receptors, JAKs/Tyk2 proximally phosphorylate them and lead to their activation to mediate downstream effector functions. For example, activated STAT6 undergoes dimerization and then translocates to the nucleus to induce critical gene transcription, conferring macrophage alternative activation and immunosuppressive functions $(3,4)$. Phosphorylated Gab2 and IRS2 initiate scaffolding of downstream molecules, leading to PI3K-Akt1/2 and other pathways that also promote immunosuppressive macrophage polarization $(5,6)$. However, the phosphorylated IL-4/13 receptors recruit not only signaling molecules potentiating an alternative phenotype, but also SH2 domain-containing tyrosine phosphatases SHP-1 and SHP-2, which dephosphorylate the receptors and temper IL-4/13 signaling. Indeed, Tachdjian et al. have shown that preventing SHP-1 from docking to IL-4R $\alpha$ (Y709) enhances IL-4/13 signaling and exacerbates allergy-associated pulmonary inflammation (7). Other studies have also shown that SHP-2 association with the IL-4 receptor suppresses macrophage alternative polarization and that inhibition of SHP-2 by a specific inhibitor PHPS1 or SHP-2 deficiency predisposes macrophages toward an anti-inflammatory phenotype (8-10).

SIRPa is a myeloid leukocyte-expressed immunoreceptor tyrosine-based inhibitory/switch motifs (ITIMs/ITSMs)-containing signaling receptor whose canonical function, via interacting with the self-marker CD47, is to inhibit professional phagocytes, e.g. macrophages and dendritic cells, from phagocytosing self-cells. Besides phagocytosis, SIRP $\alpha$ also inhibits macrophage and granulocyte inflammatory responses and dampens their proinflammatory cytokine and ROS production (11-15). Under immunosuppressive conditions, our previous study found that SIRP $\alpha$ mediates different regulation, which instead of executing inhibition, promotes macrophage alternative activation to display an anti-inflammatory phenotype $(16,17)$. The detailed mechanisms by which SIRP $\alpha$ mediates distinctive regulations under varied stimulations remain unresolved, except that the phosphorylation of SIRP $\alpha$ cytoplasmic ITIMs/ITSMs and their recruitment of SHP-1 and/or SHP-2 are likely involved $(14,16,18,19)$. However, neither the stimulatory state under which SIRP $\alpha$ recruits either SHP-1 or SHP-2 nor the regulatory ramifications arising from differential SHP-1 or SHP-2 binding has been clearly demonstrated. 
In the present study, we report that upon IL-4 and IL-13 stimulation, SIRP $\alpha$ mediates a 'dis-inhibition' mechanism that, through sequestering SHP-2, promotes macrophage polarization toward an immunosuppressive, alternative activation phenotype. This regulation is initiated by an IL-4/13-induced and Src kinase-activated Bruton's tyrosine kinase (Btk), which phosphorylates the cytoplasmic tyrosines within SIRP $\alpha$ ITIMs/ITSMs in a manner that leads to exclusive docking of SHP-2, but not SHP-1. By recruiting SHP-2, SIRP $\alpha$ depletes SHP-2 from accessing IL4/13 receptors ( $\gamma \mathrm{C}$ and IL-13R $\alpha 1$ specifically), thereby enhancing IL-4R $\alpha$ phosphorylation and downstream activation of STAT6, Gab2 and PI3K-Akt. Consistent with these findings, deficiency of SIRP $\alpha$ allows SHP-2 to freely inhibit IL-4/13 receptors and subsequently dampen macrophage alternative activation, a condition associated with hindered resolution of inflammation in a murine colitis-recovery model. Converesly, transfusion of alternatively activated macrophages expressing increased SIRP $\alpha$ restored the anti-inflammatory response, promoting post-colitis wound healing and recovery. 


\section{Results}

\section{SIRP $\alpha$ promotes IL-4- and IL-13-induced macrophage alternative activation}

To test the effect of SIRP $\alpha$ on IL-4-induced alternative activation, murine macrophages freshly isolated from the peritoneum (PEM) and derived from the bone marrow (BMDM) with and without SIRP $\alpha$ expression (WT and Sirp $\alpha^{-/}$, respectively) were treated with IL-4 in the presence or absence of mCD47.ex, a soluble murine CD47 extracellular domain that ligates $\operatorname{SIRP} \alpha$ on macrophages $(11,20)$. The same experiments were also done with human macrophages (HMM) derived from peripheral blood monocytes in the presence or absence of a human CD47 extracellular domain (hCD47.ex) $(19,21)$. Both murine and human macrophages treated with IL-4 produced significantly higher IL-10 and TGF $\beta$ when their SIRP $\alpha$ was ligated by CD47 (mCD47.ex or hCD47.ex) (Fig. 1, A-C). In contrast, SIRP $\alpha$ deficiency $\left(\mathrm{Sirp}^{-/}{ }^{-}\right)$blunted IL-4 responsiveness and markedly reduced IL-10 and TGF $\beta$ production. As expected, Sirp $\alpha^{-/}$macrophages were not affected by the presence of CD47 ligation. Aside from impacting cytokine production, CD47 ligation of SIRP $\alpha$ also enhanced the expression of CD206, arginase-1 (Arg1) and the transcription of Arg1, Ym1, Msr2 and Fizz1 in IL-4-treated WT murine macrophages, whereas SIRP $\alpha$ deficiency largely reduced these phenotypic markers (Fig. 1, D-F). Investigation of IL-4 downstream signaling confirmed that CD47-SIRP $\alpha$ ligation bolstered the activation of STAT6 and PI3K-Akt pathways, having increased phosphorylation of STAT6 (p-STAT6), Akt1 and Akt2 (p-Akt1 and p-Akt2), whereas SIRP $\alpha$ deficiency dampened IL-4-induced phosphorylation of these molecules (Fig. 1G). Similar SIRP $\alpha$-mediated regulation of IL4 signaling was also observed in bone marrow-derived dendritic cells (BMDC) with the presence or absence of SIRP $\alpha$ respectively promoting or diminishing IL-4 signal transduction (Fig. 1H).

SIRP $\alpha$ similarly enhanced the alternative activation of macrophages stimulated by IL-13, which shares the IL-4 signaling cascade via the IL-4R $\alpha / \mathrm{IL}-13 \mathrm{R} \alpha 1$ complex (22). As with IL-4 stimulation, SIRP $\alpha$ ligation by CD47 increased the secretion of IL-10 and TGF $\beta$ and the expression of CD206 in IL-13-treated macrophages, whereas SIRP $\alpha$ deficiency markedly diminished these IL-13-driven effects (Fig. 1I).

\section{IL-4/13 induce phosphorylation of SIRP $\alpha$ cytoplasmic domain and recruitment of SHP-2}

To determine the mechanism by which SIRP $\alpha$ promotes IL-4/13-induced macrophage alternative activation, we assessed SIRP $\alpha$ cytoplasmic ITIMs/ITSMs phosphorylation and their association with SHP-1 and/or SHP-2. As shown (Fig. 2, A-B), IL-4 stimulation dose-dependently and rapidly ( $<5 \mathrm{~min}$ ) induced robust tyrosine phosphorylation in SIRP $\alpha\left(\mathrm{SIRP} \alpha^{\mathrm{PY}}\right)$. Moreover, IL-4-driven phosphorylation of SIRP $\alpha$ occurred independent of extracellular ligation by CD47, albeit the latter enhanced the phosphorylation intensity. Without IL-4 stimulation, mere CD47 ligation was inefficient, inducing only weak SIRP $\alpha$ phosphorylation. These results suggest that IL-4 activates a special tyrosine kinase(s), which phosphorylates SIRP $\alpha$ ITIMs/ITSMs, and that CD47 ligation triggers a structural change in the $\operatorname{SIRP} \alpha$ cytoplasmic domain that facilitates the tyrosine phosphorylation to occur. Detecting SHP-1 and SHP-2 association with SIRP $\alpha$ by co-immunoprecipitation (IP) found that phosphorylated SIRP $\alpha$ nearly exclusively recruited SHP-2, not SHP-1, which remained in the SIRP $\alpha$-excluded post-bound fraction 
(Fig. 2, A-B). Two forms of SHP-2 were detected: one having a higher molecular weight (MW; 75kDa) that was phosphorylated (p-SHP-2 or SHP-2 ${ }^{\text {Tyr542}}$ ) and another being non-phosphorylated with lower MW (72kDa, SHP2). Only phosphorylated SHP-2 bound to SIRPa ${ }^{\mathrm{PY}}$ upon IL-4 stimulation (Fig. 2, A-B). SHP-2 phosphorylation was dose-dependently correlated with IL-4 stimulation, independent of SIRP $\alpha$ expression or CD47 ligation (Fig. $2 \mathrm{C})$.

Similar to IL-4, IL-13 stimulation induced SIRP $\alpha$ phosphorylation and association of phosphorylated SHP-2, but not SHP-1 (Fig. 2D). SIRP $\alpha^{\mathrm{PY}}$ selectively recruiting SHP-2 also occured in macrophages stimulated with IL-10 and TGF $\beta$, whereas macrophages stimulated with proinflammatory (M1) factors IFN $\gamma$ and LPS comprised phosphorylated SIRP $\alpha$ that instead associated with SHP-1 (Fig. 2D). Immunoprecipitating SHP-1 or SHP-2 confirmed that SHP-1 co-precipitated with SIRP $\alpha$ in IFN $\gamma /$ LPS-treated macrophages, while SHP-2 co-precipitated with SIRP $\alpha$ under IL-4 stimulation (Fig. 2E).

\section{Src kinase-activated Btk phosphorylates SIRPa under IL-4/13 stimulation}

Given that Lyn, a Src family tyrosine kinase, has been shown to phosphorylate ITIMs in different receptors including SIRP $\alpha(23,24)$, we questioned the role of Lyn in IL-4/13-induced SIRP $\alpha$ phosphorylation. A Lynspecific inhibitor Bafetinib (also named INNO-406) was tested; however, it showed only slight inhibition on IL4/13-induced SIRP $\alpha$ phosphorylation (Fig. 3A). Instead, Bafetinib completely eliminated the low-level SIRP $\alpha$ phosphorylation induced by CD47 ligation in the absence of IL-4/13. These results suggest that Lyn was not responsible for the robust SIRP $\alpha$ phosphorylation under IL-4/13 stimulation. Instead, Lyn appeared constitutively active with a low phosphorylation capacity. To identify the tyrosine kinase(s) activated by IL-4/13, we tested pharmacological inhibitors targeting other kinases and found that the Src family kinase (SFK) inhibitor PP1, but not PP2, and the Btk inhibitor LFM-A13, but not its non-inhibitory analog LFM-A11 (Fig. 3D), strongly inhibited SIRP $\alpha$ phosphorylation and SIRP $\alpha$ association with SHP-2 by IL-4 (Fig. 3, B-C). To validate that Btk was the kinase phosphorylating $\operatorname{SIRP} \alpha$, another Btk-specific inhibitor, Ibrutinib, was used (25). As with LFM-A13, Ibrutinib dose-dependently inhibited SIRP $\alpha$ phosphorylation and association with SHP-2 in an IL-4/13-dependent manner (Fig. 3D). Additional experiments confirmed that IL-4 and IL-13 activated Btk, which had increased phosphorylation at $\operatorname{Tyr}^{223}$ (Fig. 3E), a result consistent with reports by others $(26,27)$. Both Btk inhibitors were found to deplete Btk phosphorylation (Fig. 3F). Furthermore, inhibition of SFK by PP1 prevented Btk phosphorylation (Fig. 3F), suggesting that certain PP1-sensitive SFK mediated Btk activation under IL-4/13 stimulation (depicted in Fig. 3G).

\section{Phosphorylated SIRPa sequesters SHP-2 to disinhibit IL-4/13 signaling}

Given the finding that IL-4/13 stimulation induces SIRP $\alpha$ phosphorylation and recruitment of SHP-2, which coincidentally also binds to IL-4/13 receptors and inhibits IL-4/13 signaling, we developed a hypothesis postulating that the SIRP $\alpha$-to-SHP-2 recruitment acts as a 'disinhibition' mechanism that, by SIRP $\alpha$ sequestering 
SHP-2, physically restricts SHP-2 from accessing the IL-4/13 receptors and thereby enhances cytokine-mediated signal transduction. This hypothesis agreed with the observation that SIRP $\alpha$ promoted macrophage alternative activation. To further test this hypothesis, we examined IL-4 and IL-13 receptors for phosphorylation and association with SHP-2 following cytokine stimulation in the presence or absence of SIRP $\alpha$-mediated regulation (WT \pm mCD47.ex or Sirp $\alpha^{-/}$). As expected, IL-4 and IL-13 stimulation dose-dependently induced tyrosine phosphorylation of their heterodimeric receptor chains, IL-4R $\alpha$ and $\gamma C$ or IL-13R $\alpha 1$; however, only IL-4R $\alpha$ exhibited varied phosphorylation correlated with SIRP $\alpha$-mediated regulation. As shown (Fig. 4A), under IL-4/13 stimulation, WT macrophages ligated by CD47 (+ mCD47.ex) exhibited enhanced IL-4R $\alpha$ phosphorylation compared to macrophages without $\operatorname{CD} 47$ ligation or depleted of $\operatorname{SIRP} \alpha\left(\operatorname{Sirp} \alpha^{-/}\right)$, the latter indeed displaying diminished IL-4R $\alpha$ phosphorylation even under high-dose cytokine stimulation. The level of IL-4R $\alpha$ phosphorylation was also found to correlate with receptor-mediated downstream signaling, showing significantly increased or reduced activation of STAT6 and Akt1/2 and association of Gab2 with IL-4R $\alpha$. The co-receptor $\gamma \mathrm{C}$ or IL-13R $\alpha 1$, on the other hand, displayed minimal phosphorylation variations in the presence or absence of SIRP $\alpha$-mediated regulation. These results suggest that the phosphorylation of IL-4R $\alpha$, but not its co-receptor $\gamma \mathrm{C}$ or IL-13R $\alpha 1$, is affected by SIRP $\alpha$ and impacts downstream signaling. This agrees with previous reports showing that IL-4R $\alpha$ is the principal signaling chain whose phosphorylation provides docking of downstream molecules (22).

IP of SHP-2 and detection of its co-associated cytokine receptors found that SHP-2 associated with $\gamma \mathrm{C}$ and IL13R $\alpha 1$, but not IL-4R $\alpha$, in IL-4/13-stimulated macrophages (Fig.4B). Vice versa, IP of $\gamma \mathrm{C}$ or IL-13R $\alpha 1$ detected SHP-2 (Fig.4C). Given that the antibodies used to precipitate $\gamma \mathrm{C}$ and IL-13R $\alpha 1$ interrupt IL-4/13 receptor complexes and thus do not co-precipitate with IL-4R $\alpha$, the post-bound lysates depleted of $\gamma \mathrm{C}$ or IL-13R $\alpha 1$ were subsequently used for IP of IL-4R $\alpha$; however, these experiments failed to detect SHP-2 association. On the other hand, IP of IL-4R $\alpha$ with an antibody that maintains IL-4R $\alpha-\gamma \mathrm{C} / \mathrm{IL}-13 \mathrm{R} \alpha 1$ receptor complexes co-precipitated IL4R $\alpha, \gamma \mathrm{C}$ and/or IL-13R $\alpha$ 1, and SHP-2 (Fig.4D). Comparing different macrophages (Fig.4 C-D) revealed that SHP2 poorly bound to $\gamma \mathrm{C}$ or IL-13R $\alpha 1$ in WT macrophages with elevated SIRP $\alpha$ cytoplasmic phosphorylation (+ $\mathrm{mCD} 47 . e x)$ and that this reduction was associated with increased IL-4R $\alpha$ phosphorylation. In stark contrast, SHP2 highly associated with $\gamma \mathrm{C}$ or IL-13R $\alpha 1$ in Sirp ${ }^{-/-}$macrophages upon cytokine stimulation, and this increased $\gamma \mathrm{C} / \mathrm{IL}-13 \mathrm{R} \alpha 1$ association correlated with substantially reduced phosphorylation in IL-4R $\alpha$, leading to diminished downstream signaling. Thus, the extent to which SHP-2 bound to $\gamma \mathrm{C}$ or IL-13R $\alpha 1$ was inversely correlated with IL-4R $\alpha$ phosphorylation and IL-4R $\alpha$-mediated downstream signal activation. Together ${ }_{2}$ these results strongly supported our hypothesis that SIRP $\alpha$ sequestering SHP-2 serves to dis-inhibit IL-4/13 signaling, and also revealed that SHP-2 docks to $\gamma \mathrm{C}$ or IL-13R $\alpha 1$ while mediating dephosphorylation in IL-4R $\alpha$, which controls downstream signaling (depicted in Fig. 4B). 
In tangential experiments, we observed that IL-4/13 stimulation significantly increased SIRP $\alpha$ expression in macrophages (Fig. 4 E). However, this response occurred slower (requiring $>8 \mathrm{~h}$ ) than IL-4/13-driven induction of immunosuppressive cytokines and other phenotypic markers (generally $<6 \mathrm{~h}$ ). Nevertheless, increased SIRP $\alpha$ expression (SIRP $\alpha^{\text {high }}$ ) enhanced SIRP $\alpha$-to-SHP-2 sequestration to a greater extent, nearly depleting all SHP-2 available to inhibit the IL-4/13 signaling, thereby further sensitizing macrophages for alternative activation (Fig. 4F). Consequently, SIRP $\alpha^{\text {high }}$ macrophages, especially in the presence of CD47 ligation, produced much higher levels of IL-10 and TGF $\beta$ upon IL-4/13 stimulation (Fig. 4G). Thus, IL-4/13 signaling and SIRP $\alpha$ concertedly form a positive-feedback loop wherein: IL-4/13 increases SIRP $\alpha$ expression and thereby enhances SIRP $\alpha$ mediated disinhibition through sequestration of SHP-2, a regulation that then bolsters IL-4/13 signaling to promote macrophage alternative activation.

\section{SIRPa directly regulates neither SHP-1 nor integrin under IL-4/13 signaling}

The co-IP experiments found that SHP-1 also bound to IL-4/13 receptor complexes (Fig. 4C) and this finds was consistent with studies by the Chatila group $(7,28)$. Differing from SHP-2 that bound to $\gamma$ C or IL-13R $\alpha 1$, SHP-1 bound to IL-4R $\alpha$ and was in a manner that positively correlated with the level of IL-4R $\alpha$ phosphorylation. Though SIRP $\alpha$ did not recruit SHP-1 under IL-4/13, it affected SHP-1-to-IL-4R $\alpha$ binding through regulating SHP-2mediated dephosphorylation of IL-4R $\alpha$. As shown (Fig. 4C), increased SIRP $\alpha$ sequestration of SHP-2 (WT + mCD47.ex) elevated not only IL-4R $\alpha$ phosphorylation but also IL-4R $\alpha$-SHP-1 association, whereas SIRP $\alpha$ deficiency led to strong SHP-2-mediated dephosphorylation of IL-4R $\alpha$, diminishing SHP-1-to-IL-4R $\alpha$ binding. While the manner of SHP-1-IL-4R $\alpha$ association paralleled IL-4R $\alpha$ phosphorylation and IL-4R $\alpha$-mediated downstream signaling activation, these results did not imply that SHP-1 positively regulates IL-4/13 signaling. Further studies employing phosphatase inhibitors confirmed that SHP-1, also SHP-2, inhibited IL-4/13 signaling and subsequent cytokine production (Fig. 5A). Inhibitors targeting either SHP-1 (TPI-1 and PTP-I) or SHP-2 (PHPS1 and SHP099) were tested, all of which dose-dependently increased IL-4/13-induced IL-10 and TGF $\beta$ production. As expected, SHP-2 inhibitors profoundly affected Sirp ${ }^{-/}$macrophages but not SIRP $\alpha^{\text {high }}$ macrophages, as in the latter SHP-2 activity was largely negated by SIRP $\alpha$. Meanwhile, inhibition of SHP-1 further increased the already high levels of IL-10 and TGF $\beta$ in SIRP $\alpha^{\text {high }}$ macrophages, but was less impactful toward Sirp $\alpha^{-/-}$macrophages in which IL-4/13 signaling was dominantly controlled by SHP-2. Combining SHP-1 and SHP-2 inhibitors, however, drastically augmented cytokine production in all macrophages, surpassing that which was achieved by either inhibition.

Employing the SHP-2 inhibitor SHP099 and the Btk inhibitor Ibrutinib, we further validated the IL$4 / 13 \rightarrow$ Btk $\rightarrow$ SIRP $\alpha-S H P-2 \rightarrow$ IL-4/13 regulatory axis. Supporting our previous findings, Ibrutinib inhibited Btk activation (p-Btk), which is integral to SIRP $\alpha$ phosphorylation and SHP-2 sequestration, and dampened cytokine signaling in IL-4-stimulated macrophages (i.e., reduced p-STAT6 and p-Akt). While SHP099 did not affect Btk, its inhibition of SHP-2 resulted in increased phosphorylation of STAT6 and Akt in WT and Sirpa $\alpha^{-/-}$macrophages 
alike. The combination of Ibrutinib and SHP099 resulted in signal transduction similar to employing SHP099 alone, confirming that Btk is upstream of SIRP $\alpha$-SHP-2 sequestration (Fig. 5B). Assaying cytokine production confirmed these results, showing Ibrutinib alone dose-dependently reduced IL-10 and TGF $\beta$ in SIRP $\alpha^{+/ h i g h}$ macrophages by IL-4, whereas the combination of Ibrutinib and SHP099 led to increased cytokine production as that which occurred under SHP-2 inhibition by SHP099 (Fig. 5C).

As SIRP $\alpha$ was reported to regulate leukocyte function through its cooperation with integrin-mediated cell adhesion (29), we investigated whether SIRP $\alpha$ deficiency alters cytokine-induced macrophage adhesion/spreading and if this occurs through the $\beta 2$ integrin CD11b/CD18. Stimulation of WT and Sirp ${ }^{-/-}$macrophages induced their adhesion to the matrix, but only WT macrophages displayed an activated, broad spreading and/or elongated morphology (Fig. 5D). Comparably, IL-4-stimulated Sirpa $\alpha^{-/-}$macrophages exhibited a much less outstretched morphology suggestive of insufficient activation. Further studies confirmed that this difference in morphology was not due to the absence of SIRP $\alpha$, but rather was from SHP-2 activity-reduced IL-4 signaling, as inhibition of SHP-2 by SHP099 enhanced IL-4 signaling and restored Sirp $\alpha^{-/-}$macrophage spreading. In contrast, impairing SIRP $\alpha$-to-SHP-2 sequestration in WT macrophages by inhibition of Btk (Ibrutinib) attenuated their response to IL-4 and spreading, which was restored by concomitant inhibition of SHP-2 (Ibrutinib + SHP099) (Fig. 5D). Staining of SIRP $\alpha$ and $\mathrm{CD} 11 \mathrm{~b}$ revealed punctate patterns on WT macrophage surface, though these two proteins did not co-localize (Fig. 5E). Together, these data suggest that SIRP $\alpha$ unlikely interacts with $\beta 2$ integrin, but affects macrophage adhesion/spreading through regulating cytokine signaling.

\section{SIRP $\alpha$ deficiency impairs post-colitis wound healing by reducing IL-10 ${ }^{+}$macrophages}

Alternatively activated macrophages orchestrate protective functions under inflammatory conditions and are critical for inflammation resolution $(30,31)$. Given our finding that SIRP $\alpha$ promotes alternative activation, we questioned whether SIRP $\alpha$ deficiency in vivo would impair immunosuppressive macrophage polarization and undermine disease resolution. Experimental colitis was induced in WT and Sirp $\alpha^{-/-}$mice by feeding animals with 1-3\% dextran sodium sulfate (DSS)-containing water for 6 days, and afterward mice were given DSS-free water to allow recovery. Consistent with previous studies $(14,32)$, Sirp $\alpha^{-/-}$mice compared to WT mice developed more severe colitis under the same dose of DSS treatment (D1-D6), displaying faster body weight loss and higher fecal concentrations of lipocalin 2 (Lcn-2, a useful clinical marker of colitis) (Fig. 6A). Moreover, Sirpa $\alpha^{-/-}$mice also displayed difficulty to recover after DSS removal. As shown, compared to WT mice that passed the disease apex by D9 and restored their body weight to $>95 \%$ by D14, Sirp $\alpha^{-/-}$mice exhibited protracted colitis and did not begin to recover until D15, followed by a slow recovery that would not reach $90 \%$ of their initial body weight by D2030. Examination of cytokines found increases in circulating IL-6 but not IL-17A in Sirp $\alpha^{-/}$mice (Fig. 6C), despite that the latter has been suggested to drive colitis in other studies $(32,33)$. Inspection of resected colon tissues confirmed that Sirp $\alpha^{-/}$mice experienced worse colitis and slow wound healing, which was associated with a paucity of IL- $10^{+}$macrophages but an abundance of IL- $6^{+}$macrophages and neutrophils $\left(\mathrm{Ly}_{6} \mathrm{G}^{+}\right)$along the 
mucosal lining after DSS removal (D12) (Fig. 6 B-D). Speculating that Sirp $\alpha^{-/}$macrophage inability to undergo alternative activation underpinned the failure of inflammation resolution, we prepared IL-4-induced alternatively activated macrophages (WT/SIRP $\alpha^{\text {high }} \mathrm{BMDM} \mathrm{GFP}^{+}$) ex vivo and intravenously transfused them into colitic $\mathrm{Sirp}^{-/-}$mice. Full body bioluminescent imaging revealed that IL-4-treated BMDM readily infiltrate the inflamed intestines in Sirpo ${ }^{-/}$mice (Fig. 6E). Following two rounds of transfusion (D6, D9), Sirp ${ }^{-/-}$mice markedly improved the rate at which they recovered from acute colitis, displaying both rapid body weight increase and clearance of fecal Lcn-2 (Fig. 6F). 


\section{Discussion}

In addition to controlling macrophage phagocytosis of self-cells, SIRP $\alpha$ critically regulates macrophage responses to pro- and anti-inflammatory cues and phenotypic activation. Here, we show that macrophages, especially those with high SIRP $\alpha$ expression and extracellularly ligated by CD47, are significantly augmented for polarization by Th2 cytokines IL-4 and IL-13, exhibiting increased expressions of immunosuppressive cytokines IL-10 and TGF $\beta$ and alternative activation phenotypic markers Arg-1 and CD206. In contrast, macrophages deficient of SIRP $\alpha$ displayed an inability to undergo altenative activation due to much weakened responses to IL-4/13. Together, these studies ascertained that SIRP $\alpha$ promotes a macrophage anti-inflammatory phenotype that is conducive to tissue support and immunosuppression.

The mechanism by which SIRP $\alpha$ promotes IL-4/13-induced macrophage alternative activation revealed in this study was, however, unexpected. First, although SIRP $\alpha$ cytoplasmic ITIMs/ITSMs phosphorylation was expected to mediate the regulation, phosphorylation was triggered by cytokine stimulation but not CD47 extracellular binding. Indeed, IL-4/13 induces robust SIRP $\alpha$ phosphorylation through activating a SFK-sensitive tyrosine kinase Btk. The chain of these reactions, IL-4/13 $\rightarrow \mathrm{SFK} \rightarrow \mathrm{Btk} \rightarrow \mathrm{SIRP} \alpha$, occurs independently of CD47, albeit the latter ligating the SIRP $\alpha$ extracellular domain triggers SIRP $\alpha$ cytoplasmic structural change that facilitates the extent to which its tyrosine residues become phosphorylated. In addition to cytokine-activated Btk, Lyn also phosphorylates SIRP $\alpha$ cytoplasmic domain. However, our study revealed that Lyn plays a trivial role under cytokine stimulation, but its constitutive, low kinase activity is rather useful in maintaining macrophage homeostatic function once $\operatorname{SIRP} \alpha$ is ligated by CD47.

Second, this mechanism of IL-4/13-induced SIRP $\alpha$ regulation functions like a loop of "self-authorization" that permits IL-4/13 receptors to proceed with signal transduction. Following IL-4/13 ligation, the authority for their receptors to propogate signaling is dependent on receptor tyrosine phosphorylation, which provide phosphotyrosine $(\mathrm{pY})$ docking sites for activation of downstream signal molecules. However, phosphorylated receptors recruit not only signaling molecules but also the pY-binding inhibitory molecules SHP-1 and SHP-2, which dephosphorylate IL-4/13 receptors and diminish signal transduction. Indeed, these phosphatase-mediated inhibitory activities are strong and largely control IL-4/13 signaling. As observed in Sirp $\alpha^{-/-}$macrophages where SHP-2 is unleashed to dephosphorylate IL-4/13 receptors, $>80 \%$ of IL-4/13-mediated signaling was abated, resulting in ineffective macrophage alternative activation and IL-10 and TGF $\beta$ production. In contrast, restriction of SHP-2 by SIRP $\alpha$ enabled strong IL-4/13 signaling and high macrophage phenotypic expression of immuosuppressive cytokines. Thus, the SIRP $\alpha$-mediated sequestration of SHP-2 endorses IL-4/13 signaling. As further revealed in this study, IL-4/13 signaling is in turn upregulating $\operatorname{SIRP} \alpha$ expression (SIRP $\alpha^{\text {high }}$ ), thereby forming a self-reinforcing loop, IL-4/13 $\rightarrow \mathrm{SIRP} \alpha \rightarrow \mathrm{IL}-4 / 13$, that powerfully promotes macrophage polarization towards alternative activation. 
Third, IL-4/13 induces phosphorylation of SIRP $\alpha$ ITIMs/ITSMs that controls only SHP-2, not SHP-1. Both corroborating and departing from previous work by Tachdjian et al. (7), we found that SHP-1 also inhibits IL4/13 signaling. Different from SHP-2 that binds to the co-receptor $\gamma \mathrm{C}$ or IL-13R $\alpha 1$ and dephosphorylates the signaling chain IL-4R $\alpha$, SHP-1 directly docks to IL-4R $\alpha$ (Y709). Though SIRP $\alpha$ does not physically recruit SHP1, it impacts the degree of SHP-1 association to IL-4R $\alpha$ by affecting SHP-2-mediated dephosphorylation of IL4R $\alpha$. Presumably, when SHP-2 inhibition is strong, such as that in Sirp $\alpha^{-/}$macrophages, the role of SHP-1 is negligable, as the heavy dephosphorylation of IL-4R $\alpha$ by SHP-2 depletes SHP-1 docking. Conversely, in SIRP $\alpha^{\text {high }}$ macrophages, where SHP-2 is restricted by SIRP $\alpha$ and, as such, IL-4R $\alpha$ is less dephosphorylated, SHP-1 binding to IL-4R $\alpha$ becomes prominent and its regulatory activity controls the IL-4/13 signaling strength.

The mechanism by which SIRP $\alpha$ recruits SHP-2 but not SHP-1 under IL-4/13 stimulation remains unclear. Two possibilities are conceivable: i) Btk phosphorylates SIRP $\alpha$ cytoplasmic tyrosines in a distinct pattern that confers binding affinity for only SHP-2 and not SHP-1. Though traditionally recognizing that the SIRP $\alpha$ cytoplasmic domain contains two ITIMs (ITY $Y^{433 / 436(\text { human/murine) }} \mathrm{ADL}$ and $\mathrm{LT} Y^{474 / 477} \mathrm{ADL}$ ), it also has tyrosines residues forming two characteristic ITSMs (TE $Y^{457 / 460} \mathrm{ASI}$ and SE $Y^{500 / 501} \mathrm{ASV}$ ). These ITIMs and ITSMs may be phosphorylated by different kinases under various activation conditions, forming phosphorylation patterns with differing affinities for either SHP-1 or SHP-2. Supporting this postulation, we observed that SIRP $\alpha$ mediates cytoplasmic recruitment of SHP-1 under proinflammatory stimulations (TLR ligands, IFN $\gamma$, IL-6, etc), while recruiting SHP-2 in response to IL-4, IL-13, IL-10 or TGF $\beta$. Studies by others have also shown that ITIM and ITSM structures in different receptors are capable of binding to SHP-1 or/and SHP-2 (34-37). ii) Recruitment of SHP-1 or SHP-2 is dictated by alteration of the $\mathrm{SH} 2$ domain binding sites in these phosphatases following cytokine or other treatments. Despite their structural similarity, SHP-1 and SHP-2 are commonly found docking on different pY residues and perform distinct regulatory fiunctions. Between these two phosphatases, SHP-1 generally inhibits cellular functions after being recruited to $\mathrm{pYs}$, like those in IL-4R $\alpha$, whereas the role of SHP-2 remains debated. Despite its capability of mediating inhibitory function - for example, when binding to $\gamma \mathrm{C}$ or IL-13R $\alpha 1$ and dephsohorylating IL-4R $\alpha$ - SHP-2 'positively' regulates cell signaling when recruited by SIRP $\alpha$, as well as other molecules $(38,39)$. Perhaps, the differences between SHP-1 and SHP-2 are best examplified in animals deficient of these phosphatases $(40,41)$, although further investigation is clearly needed to completely understand these regulators.

Employing a murine colitis-recovery model, we here demonstrate that Sirp $\alpha^{-/-}$mice not only develop more severe colitis due to enhanced macrophage and neutrophil functions $(42,43)$, but also exhibit greatly delayed resolution of inflammation and wound healing after DSS was removed, a condition associated with a lack of IL-10expressing macrophages in the colon mucosa. Transfusing colitic Sirp $\alpha^{-/-}$mice with SIRP $\alpha^{\text {high }}$ alternatively activated macrophages produced ex vivo redirected inflammatory condition towards resolution, leading to rapid wound healing and tissue recovery. After intravenous administration, these alternatively activated macrophages 
especially accumulated in the inflammatory intestines where they, instead of being reprogrammed by the 'hot' environment, directed an anti-inflammatory response that led to resolution of inflammation. Thus, depite that these experiments were designed to demonstrate the impact of SIRP $\alpha$ on macrophage alternative activation in vivo, they unexpectedly revealed a glimpse into the potential of using SIRP $\alpha^{\text {high }}$ macrophages as a cellular therapy for treating inflammatory diseases. Given that many of the current endeavors mitigate diseases, such as cytokine storm syndromes associated with COVID-19 and other infections, autoimmune inflammatory conditions and cancer, through immunotherapeutic modalities, leveraging the immune system by regulating SIRP $\alpha$ and directing macrophages to favor either pro-inflammatory or anti-inflammatory activation may provide a new avenue to rectify disease conditions and awaits further exploration. 


\section{Materials and Methods}

Macrophages: Murine bone marrow-derived macrophages (BMDM) were produced by differentiation of bone marrow cells from WT and Sirp $\alpha^{-/}$mice (14) with macrophage colony stimulating factor (M-CSF)-conditioned RPMI-1640 medium with 10\% FBS for 5 days (14). Murine peritoneal macrophages (PEM) were prepared by lavaging the peritoneal cavity with PBS followed by centrifugation and adherence to cell culture plates to remove non-adherent peritoneal cells (14). Human monocyte-derived macrophages (HMM) were produced by differentiating peripheral blood mononuclear cells (PBMC) in RPMI 1640 medium containing human M-CSF (10ng/mL, Biolegend) and 10\% human platelets-free plasma for 5-7 days $(42,44)$. To prevent unwanted macrophage activation towards proinflammatory polarization, a caveat often caused by undetectable contamination, dust or unknown TLR agonists in the culture, low dosage IL-10 (2ng/ml) was sometimes added into the culture, which effectively maintained an inactive macrophage phenotype as indicated by assays detecting macrophage production of cytokines and macrophage metabolic states. Addition of IL-10 during HMM differentiation has also been described elsewhere (45). Note: Failure to control the macrophage phenotype during macrophage differentiation is the most common cause compromising studies of cell signaling and functionality induced by alternative activation stimuli. SIRP $\alpha^{\text {high }}$ macrophages were induced by IL-4 treatment $(5 \mathrm{ng} / \mathrm{ml}, 16 \mathrm{~h})$ and were allowed to rest for $>12 \mathrm{~h}$ in non-IL-4-containing media prior to subsequent treatments.

Soluble murine and human CD47 extracellular domains (mCD47.ex and hCD47.ex): The AP-tag2 plasmid containing the extracellular domain of murine CD47 (mCD47.ex) and alkaline phosphatase (AP) was a generous gift of V. Narayanan (University of Pittsburgh School of Medicine) (20). This plasmid was then reconstructed by replacing mCD47.ex with the human CD47 extracellular domain (hCD47.ex). After transfecting COS cells, recombinant $\mathrm{mCD} 47$.ex and hCD47.ex fusion proteins were produced, affinity purified and stored in PBS as previously described $(11,19-21)$. Prior to use, the binding capacities of mCD47.ex or hCD47.ex with SIRP $\alpha$ were confirmed by their binding to murine or human SIRP $\alpha$ extracellular domain fusion protein, mSIRP $\alpha . \operatorname{ex}-\mathrm{Fc}$ or hSIRP $\alpha$.ex-Fc, respectively, as well as adhesion to macrophages in a CD47-SIRP $\alpha$-dependent manner (21).

Macrophage alternative activation: IL-4 or IL-13 (Biolegend or PeproTech) was added into macrophage cultures at various concentrations to induce the M2/M2a phenotype in the presence or absence of a soluble CD47 extracellular domain (mCD47.ex or hCD47.ex). At different time points (0, 4, 10, 16 and 24h), cell-free supernatants were collected followed by ELISA to detect IL-10 and TGF $\beta$ using capture and detecting antibodies (Biolegend). Other M2 phenotype characteristics including the cell surface expressions of CD206, intracellular arginase (Arg-1) expression, and mRNA levels of Arg1, Ym1, Msr2 and Fizz1, were detected by a PE-conjugated anti-CD206 antibody (Biolegend) for flow cytometry, an anti-Arg-1 antibody (Santa Cruz Biotechnology) for western blot (WB) $(46,47)$, as well as oligonucleotide primers for real-time RT-PCR. For the last set of experiments, PCR primers were: Arg1, 5'-ctccaagccaaagtccttagag and 5'-aggagctgtcattagggacatc; Ym1, 5'agaagggagtttcaaacctggt and 5'-gtcttgctcatgtgtgtaagtga; Msr2, 5'-aaagaaagcccgagtccc and 5'- 
tgcccaaggagatagcaaga; Fizz1, 5'-ggatgccaactttgaatagg and 5'-cttcgttacagtggagggat; GAPDH, 5'tgaagcaggcatctgaggg and 5'-cgaaggtggaagagtgggag (48). In other experiments, macrophages were treated with IFN $\gamma(20 \mathrm{ng} / \mathrm{ml})$, LPS $(100 \mathrm{ng} / \mathrm{ml})$, IL-10 $(20 \mathrm{ng} / \mathrm{ml})$ or TGF $\beta(20 \mathrm{ng} / \mathrm{ml})$ to induce different phenotypes in the presence or absence of mCD47.ex.

Culture of bone marrow-derived dendritic cells (BMDCs): Bone marrow-derived dendritic cells were induced by GM-CSF (20ng/ml) as previously described (49). On day 6, floating or lightly adherent cells were harvested by gentlely washing with PBS and then pooled for subsequent experiments. After 10 days induction, the cells were ready and treated with IL-4 for further analysis.

Immunoprecipitation (IP) and WB: Murine macrophages were briefly treated with freshly prepared pervanadate $\left(2 \mathrm{mM}, 5 \mathrm{~min}, 37^{\circ} \mathrm{C}\right)$ followed by lysis in an ice-cold buffer containing $25 \mathrm{mM}$ Tris-HCl, $\mathrm{pH} 7.4,150 \mathrm{mM} \mathrm{NaCl}$, 1\% Triton X-100, protease inhibitors (Protease Inhibitor Cocktail, Sigma-Aldrich), phosphatase inhibitors (Phosphatase Inhibitor Cocktail 1 and 2, Sigma), 3mM PMSF and 2mM pervanadate. After centrifugation (12,000rpm, $5 \mathrm{~min}$ ), the clear lysates were collected. For IP, antibodies against SIRP $\alpha$ (clone P84, Biolegend), SHP-1, SHP-2, IL-4R $\alpha, \gamma$ chain or IL-13R $\alpha 1$ (all from Santa Cruz Biotechnology) were added at 5 $\mu \mathrm{g} / \mathrm{ml}$ followed by precipitation with protein $\mathrm{A} / \mathrm{G}$-Sepharose $\left(4 \mathrm{~h}, 4^{\circ} \mathrm{C}\right)$. After washing, the beads were heated at $95^{\circ} \mathrm{C}(5 \mathrm{~min})$ in SDS-PAGE sample buffer. After electrophoresis in acrylamide gels, proteins were transferred onto nitrocellulose, followed by blocking with 5\% BSA and detection for SIRP $\alpha$, SHP-1, SHP-2, IL-4R $\alpha, \gamma$ chain or IL-13R $\alpha 1$ using the same antibodies. The antibody for IP IL-4R $\alpha$ could maintain the binding of IL-4R $\alpha$ with $\gamma$ chain or IL-13R $\alpha 1$, whereas antibodies against $\gamma$ chain or IL-13R $\alpha 1$ caused the dissociation of IL-4R $\alpha$ with $\gamma \mathrm{C}$ or IL-13R $\alpha 1$. Protein tyrosine phosphorylation was detected by PY20 (Biolegend) or 4G10 (Sigma), and phospho-SHP-2 was detected with an anti-phospho-SHP-2 (Tyr542) antibody (Cell Signaling Technology). Alternatively, macrophage lysates prior to IP or post-bound of IP were applied to SDS-PAGE and WB to detect the same proteins, as well as signal transduction molecules including Gab2, STAT6 and phospho-STAT6 (Tyr641), Akt1 and phospho-Akt1 (Ser473), Akt2 and phospho-Akt2 (Ser474), Akt and phospho-Akt (Ser473), Btk and phospho-Btk (Tyr223) (all antibody reagents from Cell Signaling Technology). The densitometry was done using the NIH software Image J.

Inhibitor treatment: To inhibit SHP-2, specific inhibitors PHPS1 (Cayman Chemical, IC50=2.1 $\mu \mathrm{M}$ ) or SHP099 (Cayman Chemical, IC50 $=71 \mathrm{nM}$ ) were used to treat macrophages prior to IL-4-induced alternative activation (8). To inhibit SHP-1, specific inhibitors TPI-1 (Cayman Chemical, IC50 = 40nM) or PTP Inhibitor I (PTP-I, Cayman Chemical, $\mathrm{K}_{\mathrm{i}}=43 \mu \mathrm{M}$ ) were used to treat macrophages before IL-4 induction. To inhibit Lyn, a Lynspecific inhibitor Bafetinib ( 5 or $10 \mu \mathrm{M}$, Cayman Chemical, IC50 = 19nM)) was used to treat macrophages with or without ligation by mCD47.ex and/or treatment by IL-4 (23). To identify other tyrosine kinase(s) that phosphorylate SIRP $\alpha$, the IL-4-stimulated macrophages were treated with the Src family kinase inhibitor PP1 $(40 \mu \mathrm{M}, \mathrm{IC} 50=170 \mathrm{nM})$ or PP2 $(20 \mu \mathrm{M}, \mathrm{IC} 50=100 \mathrm{nM})$, the pan-JAK inhibitor JAK inhibitor I $(100 \mathrm{nM}, \mathrm{IC} 50=$ 
$15 \mathrm{nM})$, the Btk inhibitor Ibrutinib $(0.05,0.5$ and $5 \mu \mathrm{M}$, IC50 =0.5nM) or LFM-A13 $(50,100$ and $150 \mu \mathrm{M}$, IC50= $2.5 \mu \mathrm{M})$, or Syk inhibitor piceatannol $(40 \mu \mathrm{M}$, IC50 = 10 $\mu \mathrm{M})$ (all from Cayman Chemical) for 20min, prior to treating with pervanadate and cell lysis (50-52). Subsequent IP and WB were performed to detect SIRP $\alpha$

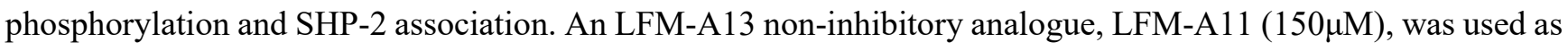
a control.

DSS-induced colitis: To induce colitis, WT mice and Sirp $\alpha^{-/}$mice were treated with 1\% DSS, $2 \%$ DSS or 3\% DSS in drinking water for 6 days, followed by removal of DSS to allow recovery. To detect lipocalin 2 (Lcn-2) in feces, fecal samples were prepared in PBS with $0.1 \%$ Tween $20(100 \mathrm{mg} / \mathrm{ml})$ and were centrifuged to obtain clear supernatants, in which Lcn-2 levels were assayed using a murine Lcn-2 ELISA kit (R\&D Systems). For macrophage adoptive transfer, GFP ${ }^{+}$WT BMDM were treated with IL-4 $(5 \mathrm{ng} / \mathrm{ml})$ for $16 \mathrm{~h}$. The Sirp $\alpha^{-/-}$mice were intravenously transfused with IL-4 activated BMDM $\left(1 \times 10^{7}\right) 2 \mathrm{~h}$ after removal of DSS (D6) at three day intervals for a total of two treatments.

Immunofluorescent tissue staining: DSS-treated mice were euthanized followed by harvesting of intestines. Intestines frozen in Tissue-Tek OCT were cryosectioned to 5-10 $\mu \mathrm{m}$ slides, which were then fixed in ethanol and blocked with PBS containing 1\% BSA (Sigma). H\&E staining was performed to determine disease conditions. For immunofluorescence staining, slides were stained with rat anti-mouse IL-10, rat anti-mouse F4/80, rat antimouse IL-6 or rat anti-mouse Ly6G (all from Biolegend), followed by fluorescence-conjugated secondary antibodies. After washing, slides were mounted with DAPI (Invitrogen) and analyzed by fluorescent microscopy.

Statistical analysis: All results represent at least three independent experiments. Quantitative data were analyzed in GraphPad Prism and are presented as means \pm SEM, and differences were considered to be statistically significant when $\mathrm{p}<0.05$ by the Student's t tests for paired samples. For multiple pairwise comparisons between samples and a shared control, analysis of two-way ANOVA with Tukey's post hoc test was performed.

\section{Acknowledgments}

This work was supported, in part, by the NIH grants AI106839 and CA241271, the Biolocity Innovation program and a venture development grant from the Georgia Research Alliance (GRA).

\section{Author contributions}

Y.L. initiated the study and oversaw all data acquisition and analysis. L.S. performed all the key experiments with critical help from Z.B., K.K., S.C. and C.O.. L.S., K.K. and Y.L. wrote the manuscript.

\section{Conflict of interest}

The authors have no conflicting interest to claim. 


\section{References}

1. A. Shapouri-Moghaddam, S. Mohammadian, H. Vazini, M. Taghadosi, S. A. Esmaeili, F. Mardani, B. Seifi, A. Mohammadi, J. T. Afshari, A. Sahebkar, Macrophage plasticity, polarization, and function in health and disease. J. Cell. Physiol. 233, 6425-6440 (2018).

2. P. J. Murray, J. E. Allen, S. K. Biswas, E. A. Fisher, D. W. Gilroy, S. Goerdt, S. Gordon, J. A. Hamilton, L. B. Ivashkiv, T. Lawrence, M. Locati, A. Mantovani, F. O. Martinez, J. L. Mege, D. M. Mosser, G. Natoli, J. P. Saeij, J. L. Schultze, K. A. Shirey, A. Sica, J. Suttles, I. Udalova, J. A. van Ginderachter, S. N. Vogel, T. A. Wynn, Macrophage activation and polarization: nomenclature and experimental guidelines. Immunity 41, 14-20 (2014).

3. S. J. Van Dyken, R. M. Locksley, Interleukin-4- and interleukin-13-mediated alternatively activated macrophages: roles in homeostasis and disease. Annu. Rev. Immunol. 31, 317-343 (2013).

4. Z. Ul-Haq, S. Naz, M. A. Mesaik, Interleukin-4 receptor signaling and its binding mechanism: A therapeutic insight from inhibitors tool box. Cytokine Growth Factor Rev. 32, 3-15 (2016).

5. X. Guo, T. Li, Y. Xu, X. Xu, Z. Zhu, Y. Zhang, J. Xu, K. Xu, H. Cheng, X. Zhang, Y. Ke, Increased levels of Gab1 and Gab2 adaptor proteins skew interleukin-4 (IL-4) signaling toward M2 macrophage-driven pulmonary fibrosis in mice. J. Biol. Chem. 292, 14003-14015 (2017).

6. E. Vergadi, E. Ieronymaki, K. Lyroni, K. Vaporidi, C. Tsatsanis, Akt Signaling Pathway in Macrophage Activation and M1/M2 Polarization. J. Immunol. 198, 1006-1014 (2017).

7. R. Tachdjian, S. Al Khatib, A. Schwinglshackl, H. S. Kim, A. Chen, J. Blasioli, C. Mathias, H. Y. Kim, D. T. Umetsu, H. C. Oettgen, T. A. Chatila, In vivo regulation of the allergic response by the IL-4 receptor alpha chain immunoreceptor tyrosine-based inhibitory motif. J. Allergy Clin. Immunol. 125, 1128-1136 (2010).

8. B. Tao, W. Jin, J. Xu, Z. Liang, J. Yao, Y. Zhang, K. Wang, H. Cheng, X. Zhang, Y. Ke, Myeloid-specific disruption of tyrosine phosphatase Shp2 promotes alternative activation of macrophages and predisposes mice to pulmonary fibrosis. J. Immunol. 193, 2801-2811 (2014).

9. L. Zhao, J. Xia, T. Li, H. Zhou, W. Ouyang, Z. Hong, Y. Ke, J. Qian, F. Xu, Shp2 Deficiency Impairs the Inflammatory Response Against Haemophilus influenzae by Regulating Macrophage Polarization. The Journal of Infectious Diseases 214, 625-633 (2016).

10. S. S. Wang, Y. Y. Yao, H. X. Li, G. Zheng, S. Lu, W. B. Chen, Tumor-associated macrophages (TAMs) depend on Shp2 for their anti-tumor roles in colorectal cancer. Am. J. Cancer Res. 9, 1957-+ (2019).

11. Y. Liu, H. J. Buhring, K. Zen, S. L. Burst, F. J. Schnell, I. R. Williams, C. A. Parkos, Signal regulatory protein (SIRPalpha), a cellular ligand for CD47, regulates neutrophil transmigration. J. Biol. Chem. 277, 1002810036 (2002).

12. Y. Liu, M. B. O'Connor, K. J. Mandell, K. Zen, A. Ullrich, H. J. Buhring, C. A. Parkos, Peptide-mediated inhibition of neutrophil transmigration by blocking CD47 interactions with signal regulatory protein alpha. $J$. Immunol. 172, 2578-2585 (2004).

13. K. Zen, Y. Guo, Z. Bian, Z. Lv, D. Zhu, H. Ohnishi, T. Matozaki, Y. Liu, Inflammation-induced proteolytic processing of the SIRPalpha cytoplasmic ITIM in neutrophils propagates a proinflammatory state. Nat Commun 4, 2436 (2013).

14. Z. Bian, L. Shi, Y. L. Guo, Z. Lv, C. Tang, S. Niu, A. Tremblay, M. Venkataramani, C. Culpepper, L. Li, Z. Zhou, A. Mansour, Y. Zhang, A. Gewirtz, K. Kidder, K. Zen, Y. Liu, Cd47-Sirpalpha interaction and IL-10 constrain inflammation-induced macrophage phagocytosis of healthy self-cells. Proc. Natl. Acad. Sci. U. S. A. 113, E5434-5443 (2016).

15. E. M. van Beek, J. A. Zarate, R. van Bruggen, K. Schornagel, A. T. Tool, T. Matozaki, G. Kraal, D. Roos, T. K. van den Berg, SIRPalpha controls the activity of the phagocyte NADPH oxidase by restricting the expression of gp91(phox). Cell Rep. 2, 748-755 (2012).

16. L. Shi, T. Bian, Y. Liu, Dual role of SIRP alpha in macrophage activation: inhibiting M1 while promoting M2 polarization via selectively activating SHP-1 and SHP-2 signal. J. Immunol. 198, (2017).

17. Y. Lin, J. L. Zhao, Q. J. Zheng, X. Jiang, J. Tian, S. Q. Liang, H. W. Guo, H. Y. Qin, Y. M. Liang, H. Han, Notch Signaling Modulates Macrophage Polarization and Phagocytosis Through Direct Suppression of Signal Regulatory Protein a Expression. Front. Immunol. 9, (2018).

18. T. Matozaki, Y. Murata, H. Okazawa, H. Ohnish, Functions and molecular mechanisms of the CD47SIRP alpha signalling pathway. Trends Cell Biol. 19, 72-80 (2009). 
19. Z. Lv, Z. Bian, L. Shi, S. Niu, B. Ha, A. Tremblay, L. Li, X. Zhang, J. Paluszynski, M. Liu, K. Zen, Y. Liu, Loss of Cell Surface CD47 Clustering Formation and Binding Avidity to SIRPalpha Facilitate Apoptotic Cell Clearance by Macrophages. J. Immunol. 195, 661-671 (2015).

20. P. H. Jiang, C. F. Lagenaur, V. Narayanan, Integrin-associated protein is a ligand for the P84 neural adhesion molecule. J. Biol. Chem. 274, 559-562 (1999).

21. Y. Liu, Q. Tong, Y. Zhou, H. W. Lee, J. J. Yang, H. J. Buhring, Y. T. Chen, B. Ha, C. X. Chen, Y. Yang, K. Zen, Functional elements on SIRPalpha IgV domain mediate cell surface binding to CD47. J. Mol. Biol. 365, 680-693 (2007).

22. S. M. McCormick, N. M. Heller, Commentary: IL-4 and IL-13 receptors and signaling. Cytokine 75, 3850 (2015).

23. R. J. Cornall, J. G. Cyster, M. L. Hibbs, A. R. Dunn, K. L. Otipoby, E. A. Clark, C. C. Goodnow, Polygenic autoimmune traits: Lyn, CD22, and SHP-1 are limiting elements of a biochemical pathway regulating BCR signaling and selection. Immunity 8, 497-508 (1998).

24. H. Nishizumi, K. Horikawa, I. Mlinaric-Rascan, T. Yamamoto, A double-edged kinase Lyn: a positive and negative regulator for antigen receptor-mediated signals. J. Exp. Med. 187, 1343-1348 (1998).

25. T. Munir, J. R. Brown, S. O'Brien, J. C. Barrientos, P. M. Barr, N. M. Reddy, S. Coutre, C. S. Tam, S. P. Mulligan, U. Jaeger, T. J. Kipps, C. Moreno, M. Montillo, J. A. Burger, J. C. Byrd, P. Hillmen, S. Dai, A. Szoke, J. P. Dean, J. A. Woyach, Final analysis from RESONATE: Up to six years of follow-up on ibrutinib in patients with previously treated chronic lymphocytic leukemia or small lymphocytic lymphoma. Am. J. Hematol. 94, 13531363 (2019).

26. S. E. M. Herman, A. L. Gordon, E. Hertlein, A. Ramanunni, X. L. Zhang, S. Jaglowski, J. Flynn, J. Jones, K. A. Blum, J. J. Buggy, A. Hamdy, A. J. Johnson, J. C. Byrd, Bruton tyrosine kinase represents a promising therapeutic target for treatment of chronic lymphocytic leukemia and is effectively targeted by PCI-32765. Blood 117, 6287-6296 (2011).

27. A. J. Gunderson, M. M. Kaneda, T. Tsujikawa, A. V. Nguyen, N. I. Affara, B. Ruffell, S. Gorjestani, S. M. Liudahl, M. Truitt, P. Olson, G. Kim, D. Hanahan, M. A. Tempero, B. Sheppard, B. Irving, B. Y. Chang, J. A. Varner, L. M. Coussens, Bruton Tyrosine Kinase-Dependent Immune Cell Cross-talk Drives Pancreas Cancer. Cancer Discov. 6, 270-285 (2016).

28. G. K. K. Hershey, M. F. Friedrich, L. A. Esswein, M. L. Thomas, T. A. Chatila, The association of atopy with a gain-of-function mutation in the alpha subunit of the interleukin-4 receptor. N. Engl. J. Med. 337, 17201725 (1997).

29. F. J. Alenghat, Q. J. Baca, N. T. Rubin, L. I. Pao, T. Matozaki, C. A. Lowell, D. E. Golan, B. G. Neel, K. D. Swanson, Macrophages require Skap2 and Sirpalpha for integrin-stimulated cytoskeletal rearrangement. J. Cell Sci. 125, 5535-5545 (2012).

30. Y. Lin, X. Yang, W. Yue, X. Xu, B. Li, L. Zou, R. He, Chemerin aggravates DSS-induced colitis by suppressing M2 macrophage polarization. Cell. Mol. Immunol. 11, 355-366 (2014).

31. M. M. Hunter, A. Wang, K. S. Parhar, M. J. Johnston, N. Van Rooijen, P. L. Beck, D. M. McKay, In vitro-derived alternatively activated macrophages reduce colonic inflammation in mice. Gastroenterology $\mathbf{1 3 8}$, 1395-1405 (2010).

32. W. O'Connor, L. A. Zenewicz, R. A. Flavell, The dual nature of $\mathrm{T}(\mathrm{H}) 17$ cells: shifting the focus to function. Nat. Immunol. 11, 471-476 (2010).

33. Z. Bian, Y. Guo, B. Ha, K. Zen, Y. Liu, Regulation of the inflammatory response: enhancing neutrophil infiltration under chronic inflammatory conditions. J. Immunol. 188, 844-853 (2012).

34. P. Scapini, S. Pereira, H. Zhang, C. A. Lowell, Multiple roles of Lyn kinase in myeloid cell signaling and function. Immunol. Rev. 228, 23-40 (2009).

35. B. E. Tourdot, M. K. Brenner, K. C. Keough, T. Holyst, P. J. Newman, D. K. Newman, Immunoreceptor Tyrosine-Based Inhibitory Motif (ITIM)-Mediated Inhibitory Signaling Is Regulated by Sequential Phosphorylation Mediated by Distinct Nonreceptor Tyrosine Kinases: A Case Study Involving PECAM-1. Biochemistry 52, 2597-2608 (2013).

36. Q. Zhang, W. B. Lee, J. S. Kang, L. K. Kim, Y. J. Kim, Integrin CD11b negatively regulates Mincleinduced signaling via the Lyn-SIRPalpha-SHP1 complex. Exp. Mol. Med. 50, e439 (2018).

37. J. M. Chemnitz, R. V. Parry, K. E. Nichols, C. H. June, J. L. Riley, SHP-1 and SHP-2 associate with immunoreceptor tyrosine-based switch motif of programmed death 1 upon primary human $\mathrm{T}$ cell stimulation, but only receptor ligation prevents T cell activation. J. Immunol. 173, 945-954 (2004). 
38. X. J. Li, C. B. Goodwin, S. C. Nabinger, B. M. Richine, Z. Yang, H. Hanenberg, H. Ohnishi, T. Matozaki, G. S. Feng, R. J. Chan, Protein-tyrosine phosphatase Shp2 positively regulates macrophage oxidative burst. $J$. Biol. Chem. 290, 3894-3909 (2015).

39. C. Niogret, W. Birchmeier, G. Guarda, SHP-2 in Lymphocytes' Cytokine and Inhibitory Receptor Signaling. Front. Immunol. 10, 2468 (2019).

40. Z. Z. Chong, K. Maiese, The Src homology 2 domain tyrosine phosphatases SHP-1 and SHP-2: diversified control of cell growth, inflammation, and injury. Histol. Histopathol. 22, 1251-1267 (2007).

41. U. Lorenz, SHP-1 and SHP-2 in T cells: two phosphatases functioning at many levels. Immunol. Rev. 228, 342-359 (2009).

42. K. Zen, Y. Guo, Z. Bian, Z. Lv, D. Zhu, H. Ohnishi, T. Matozaki, Y. Liu, Inflammation-induced proteolytic processing of the SIRPalpha cytoplasmic ITIM in neutrophils propagates a proinflammatory state. Nature Communications 4, 2436 (2013).

43. K. Kidder, Z. Bian, L. Shi, Y. Liu, Inflammation Unrestrained by SIRPalpha Induces Secondary Hemophagocytic Lymphohistiocytosis Independent of IFN-gamma. J. Immunol. 205, 2821-2833 (2020).

44. D. H. Zhu, C. Y. Pan, L. M. Li, Z. Bian, Z. Y. Lv, L. Shi, J. Zhang, D. H. Li, H. W. Gu, C. Y. Zhang, Y. Liu, K. Zen, MicroRNA-17/20a/106a modulate macrophage inflammatory responses through targeting signalregulatory protein alpha. J. Allergy Clin. Immunol. 132, 426-+ (2013).

45. X. Jin, H. S. Kruth, Culture of Macrophage Colony-stimulating Factor Differentiated Human Monocytederived Macrophages. Journal of Visualized Experiments, (2016).

46. Z. Bian, A. M. Abdelaal, L. Shi, H. W. Liang, L. Q. Xiong, K. Kidder, M. Venkataramani, C. Culpepper, K. Zen, Y. Liu, Arginase-1 is neither constitutively expressed in nor required for myeloid-derived suppressor cellmediated inhibition of T-cell proliferation. Eur. J. Immunol. 48, 1046-1058 (2018).

47. Z. Bian, L. Shi, M. Venkataramani, A. M. Abdelaal, C. Culpepper, K. Kidder, H. Liang, K. Zen, Y. Liu, Tumor conditions induce bone marrow expansion of granulocytic, but not monocytic, immunosuppressive leukocytes with increased CXCR2 expression in mice. Eur. J. Immunol. 48, 532-542 (2018).

48. X. Cai, Y. Yin, N. Li, D. Zhu, J. Zhang, C. Y. Zhang, K. Zen, Re-polarization of tumor-associated macrophages to pro-inflammatory M1 macrophages by microRNA-155. J. Mol. Cell. Biol. 4, 341-343 (2012).

49. A. Maroof, M. Penny, R. Kingston, C. Murray, S. Islam, P. A. Bedford, S. C. Knight, Interleukin-4 can induce interleukin-4 production in dendritic cells. Immunology 117, 271-279 (2006).

50. K. Zen, Y. Liu, Role of different protein tyrosine kinases in fMLP-induced neutrophil transmigration. Immunobiology 213, 13-23 (2008).

51. B. Lu, T. Nakamura, K. Inouye, J. Li, Y. Tang, P. Lundback, S. I. Valdes-Ferrer, P. S. Olofsson, T. Kalb, J. Roth, Y. Zou, H. Erlandsson-Harris, H. Yang, J. P. Ting, H. Wang, U. Andersson, D. J. Antoine, S. S. Chavan, G. S. Hotamisligil, K. J. Tracey, Novel role of PKR in inflammasome activation and HMGB1 release. Nature 488, 670-674 (2012).

52. A. Colado, M. Genoula, C. Cougoule, J. L. Marin Franco, M. B. Almejun, D. Risnik, D. Kviatcovsky, E. Podaza, E. E. Elias, F. Fuentes, I. Maridonneau-Parini, F. R. Bezares, H. Fernandez Grecco, M. Cabrejo, C. Jancic, M. D. C. Sasiain, M. Giordano, R. Gamberale, L. Balboa, M. Borge, Effect of the BTK inhibitor ibrutinib on macrophage- and gammadelta T cell-mediated response against Mycobacterium tuberculosis. Blood Cancer J. 8, 100 (2018).

53. P. C. Cook, L. H. Jones, S. J. Jenkins, T. A. Wynn, J. E. Allen, A. S. MacDonald, Alternatively activated dendritic cells regulate CD4(+) T-cell polarization in vitro and in vivo. Proc. Natl. Acad. Sci. U. S. A. 109, $9977-$ 9982 (2012). 


\section{Figure legend}

Figure 1. SIRP $\alpha$ promotes IL-4/13-induced macrophage alternative activation. (A) WT and Sirp $\alpha^{-/-}$BMDM were treated with IL-4 for indicated time periods or by various doses of IL-4 in the presence or absence of mCD47.ex, and the levels of IL-10 and TGF $\beta$ in the supernatants were tested by ELISA. (B) PEM from WT and Sirp $\alpha^{-/-}$mice were also activated by IL-4 $(2 \mathrm{ng} / \mathrm{ml})$ in the presence or absence of mCD47.ex, followed by analysis of IL-10 and TGF $\beta$ secretion after 16h. (C) The production of IL-10 and TGF $\beta$ by IL-4-treated human PBMC-derived macrophages (HMM) in the presence or absence of hCD47.ex. (D) The expression of CD206 in WT and Sirpa ${ }^{-/-}$ macrophages detected by flow cytometry. (E) The level of Arg-1 in WT and Sirpa ${ }^{-/}$BMDM determined by WB. (F) The transcription of Arg1, Ym1, Msr2 and Fizz1 in WT and Sirp $\alpha^{-/}$BMDM examined by RT-PCR. (G) The phosphorylation of STAT6, Akt1 and Akt2 in WT and Sirpo ${ }^{-/-}$BMDM during IL-4-induced activation. The phosphorylation of STAT6, Akt1 and Akt2 was quantified by densitometric analysis and normalized against the corresponding total protein. (H) WT and Sirp ${ }^{-/-}$BMDC were treated with various doses of IL-4 for $2 \mathrm{~h}$ in the presence or absence of mCD47.ex. The levels of biologically active (released) IL-4 in fresh complete medium were tested by ELISA and the expression of CD206 in IL-4 (10ng/ml)-treated WT and Sirpa $\alpha^{-/-}$BMDC after $16 \mathrm{~h}$ $(49,53)$. (I) The production of IL-10 and TGF $\beta$ by various doses of IL-13-treated BMDM in the presence or absence of mCD47.ex and the expression of CD206 in IL-13 (10ng/ml)-treated WT and Sirpa ${ }^{-/-}$macrophages detected by flow cytometry. Data are presented as mean \pm SEM of at least three independent experiments $(n \geq 3)$. Statistical significance was determined by two-way ANOVA with Tukey's post hoc test (A, G, H, I) or Student's $\mathrm{t}$ test $(\mathbf{B}, \mathbf{C}, \mathbf{D}, \mathbf{F}, \mathbf{H}, \mathbf{I})$, where ${ }^{* *} \mathrm{p}<0.01$ and $* * * \mathrm{p}<0.001$. All blots are representative of at least three independent experiments.

Figure 2. IL-4/13 induces phosphorylation of SIRP $\alpha$ cytoplasmic domain and the recruitment of SHP-2. (A) WT BMDM were treated with various doses of IL-4 for $30 \mathrm{~min}$ in the presence of mCD47.ex. SIRP $\alpha$ phosphorylation and SHP-1/2 association were assessed following IP of SIRP $\alpha$. (B) WT BMDM were treated with IL-4 (5ng/ml) for 5 or $20 \mathrm{~min}$ in the presence or absence of $\mathrm{mCD} 47$.ex. SIRP $\alpha$ phosphorylation and its binding to SHP-1/2 were assessed following IP of SIRP $\alpha$. (C) Dose-dependent effect of IL-4 on phosphorylation of SHP-2. Note: IL-4 dose-dependently enhances the level of phosphorylation of SHP-2 on Tyr542 and SIRP $\alpha$ signaling has no effect on SHP-2 phosphorylation. (D) WT BMDM were treated with IFN $\gamma(20 \mathrm{ng} / \mathrm{ml})$, LPS (100ng/ml), IL-4 (5ng/ml), IL-10 $(20 \mathrm{ng} / \mathrm{ml})$, TGF $\beta(20 \mathrm{ng} / \mathrm{ml})$ or various doses of IL-13 in the presence of mCD47.ex for 20min and the association of SHP-1 and SHP-2 with SIRP $\alpha$ was detected by IP of SIRP $\alpha$. (E) WT BMDM were treated with IFN $\gamma(20 \mathrm{ng} / \mathrm{ml}) / \mathrm{LPS}(100 \mathrm{ng} / \mathrm{ml})$ or IL-4 $(5 \mathrm{ng} / \mathrm{ml})$ for $20 \mathrm{~min}$ and the selective association of SIRP $\alpha$ with SHP-1 or SHP-2 was also detected and quantified by IP of SHP-1 or SHP-2. Meanwhile, macrophages were treated with $\mathrm{mCD} 47$.ex to ligate SIRP $\alpha$ during both activations. Data are presented as mean $\pm \mathrm{SEM}$ of at least three independent experiments $(n \geq 3)$. Statistical significance was determined by Student's t test $(\mathbf{A})$ or two-way ANOVA with Tukey's post hoc test $(\mathbf{A}, \mathbf{B})$ where ${ }^{* * *} \mathrm{p}<0.001$. All blots are representative of at least three independent experiments. 
Figure 3. Src kinase-activated Btk phosphorylates SIRP $\alpha$ under IL-4/13 stimulation. Macrophages were activated by IL-4 $(5 \mathrm{ng} / \mathrm{ml})$ in the presence of $\mathrm{mCD} 47$.ex for $20 \mathrm{~min}$ to initiate downstream signaling of JAK, followed by inhibition of different tyrosine kinases using inhibitors. (A) Does-dependent effect of Bafetinib on SIRP $\alpha$ phosphorylation and its binding to SHP-2 in mCD47.ex and/or IL-4 (5ng/ml)-treated macrophages. (B) Effect of various tyrosine kinase inhibitors on SIRP $\alpha$ phosphorylation and SHP-2 association in IL-4 (5ng/ml)-activated macrophages. Data are presented as mean \pm SEM of at least three independent experiments $(n \geq 3)$. Statistical significance was determined by Student's t test where ***p $<0.001$. (C) Dose-dependent effect of Btk inhibitor LFM-A13 or Ibrutinib on SIRP $\alpha$ phosphorylation and SHP-2 recruitment in IL-4-treated macrophages. Inactive LFM-A11 served as a negative control. (D) The phosphorylation of Btk after IL-4 (5ng/ml) or IL-13 (5ng/ml) treatment for $30 \mathrm{~min}$. (E) Effect of different tyrosine kinase inhibitors on the phosphorylation of Btk in IL-4 activated macrophages. (F) Schematic depiction of the role of Btk in SIRP $\alpha$ phosphorylation and SHP-2 recruitment during IL-4-stimulated macrophage activation. All blots represent at least three independent experiments $(n \geq 3)$.

Figure 4. Phosphorylated SIRP $\alpha$ sequesters SHP-2 to disinhibit IL-4/13 signaling. (A) WT and Sirp ${ }^{-/-}$BMDM were treated with IL-4/13 ( 2 or $20 \mathrm{ng} / \mathrm{ml}$ each) in the presence or absence of mCD47.ex for $20 \mathrm{~min}$. The phosphorylation of IL-4R $\alpha, \gamma$ chain or IL-13R $\alpha 1$ was detected by IP of IL-4R $\alpha, \gamma$ chain or IL-13R $\alpha 1$, respectively. The Gab2 associated with IL-4R $\alpha$ and the phosphorylation of STAT6 and Akt in WT and Sirp $\alpha^{-/-}$BMDM was also detected. (B) The binding of SHP-2 to IL-4R $\alpha, \gamma$ chain or IL-13R $\alpha 1$ in WT and Sirp $\alpha^{-/-}$BMDM after IL-4/13 (10ng/ml each) treatment was detected by IP of SHP-2. (C) Sequential IP of $\gamma$ chain, IL-13R $\alpha 1$ and IL-4R $\alpha$ from total cell lysate followed by WB of $\gamma$ chain $^{\text {PY }}$, IL-13R $\alpha 1^{\text {PY }}$, IL-4R $\alpha^{\text {PY }}$, SHP-1, SHP-2 and Gab2. (D) The IL-4/13 receptor complexes were analyzed by IP of IL-4R $\alpha$. (E) Time course of SIRP $\alpha$ expression in WT BMDM after IL-4 (5ng/ml) or IL-13 (5ng/ml) and dose-dependent regulation of SIRP $\alpha$ by IL-4 after 24h. (F) WT, SIRP $\alpha^{\text {high }}$ or Sirp $\alpha^{-/-}$BMDM were treated with IL-4/13 (10ng/ml each) and the association of SHP-2 with SIRP $\alpha, \gamma$ chain or IL-13R $\alpha 1$ were assessed by IP of SIRP $\alpha, \gamma$ chain or IL-13R $\alpha 1$, respectively. (G) The production of IL-10 and TGF $\beta$ from WT $\left(\mathrm{SIRP}^{+}\right)$, SIRP $\alpha^{\text {high }}$ and Sirp $\alpha^{-/-}$macrophages in response to IL-4/13 (10ng/ml) after 24h. Data were presented as mean \pm SEM of at least three independent experiments $(n \geq 3)$. Statistical significance was determined by Student's $t$ test $(\mathbf{A}, \mathbf{E}, \mathbf{G})$ or where $* \mathrm{p}<0.05, * * \mathrm{p}<0.01$ and $* * * \mathrm{p}<0.001$. All blots are representative of at least three independent experiments.

Figure 5. SHP-2 inhibition restores IL-4 signaling in SIRP $\alpha$-deficient macrophages whereas Btk inhibition diminishes IL-4 signals in WT macrophages. (A) Effect of dose-dependent inhibition of SHP-1 (TPI-1 (0.005, 0.05 and $0.5 \mu \mathrm{M})$ or PTP-I $(1,10$ and $100 \mu \mathrm{M}))$, SHP-2 (PHS1 $(0.5,5$ and $50 \mu \mathrm{M})$ or SHP099 $(0.01,0.1$ and $1 \mu \mathrm{M}))$ or both on the production of IL-10 (upper) and TGF $\beta$ (lower) in macrophages treated by IL-4/13 (5ng/ml, each) for 16h. (B) Effect of Btk inhibitor, SHP-2 inhibitor or the combination on IL-4-induced phosphorylation of STAT6 and Akt in WT and Sirp $\alpha^{-/-}$macrophages. (C) Effect of dose-dependent inhibition of SHP-2 (SHP099) or 
Btk (Ibrutinib) on the production of IL-10 (upper) and TGF $\beta$ (lower) in macrophages treated by IL-4/13 (5ng/ml, each) for $16 \mathrm{~h}$. Data were presented as mean \pm SEM of at least three independent experiments $(n \geq 3)$. Statistical significance was determined by Student's t test (A, C) where $* \mathrm{p}<0.05, * * \mathrm{p}<0.01$ and $* * * \mathrm{p}<0.001$. (D) Immunostaining of CD1 $1 \mathrm{~b}$ on WT and Sirp ${ }^{-/-}$PEM in response to IL-4 $(5 \mathrm{ng} / \mathrm{ml})$ or IL-13 $(5 \mathrm{ng} / \mathrm{ml})$ in the presence of Btk inhibitor, SHP-2 inhibitor or both. Scale bar $=10 \mu \mathrm{m}$. (E) Immunostaining of CD11b and SIRP $\alpha$ on WT PEM. Scale bar $=10 \mu \mathrm{m}$. All blots are representative of at least three independent experiments.

Figure 6. SIRP $\alpha$ deficiency impairs recovery from acute colitis in mice. (A-D) WT or Sirp $\alpha^{-/-}$mice were treated with $1 \%, 2 \%$ or $3 \%$ DSS to induce acute colitis (D1-6), followed by allowing recovery (D6-21). Development of colitis was monitored by body weight loss and fecal Len-2 (A) and shortened intestines (D12, B). The levels of IL-17 and IL-6 in serum (C) and colon sections showing infiltration of IL-10- and IL-6-expressing macrophages (red and green, respectively) and neutrophil $\left(\mathrm{Ly}_{6} \mathrm{G}^{+}\right.$, red) into intestines (D) were measured. Scale bar $=50 \mu \mathrm{m}$. (E-F) 2\% DSS-induced colitic Sirp $\alpha^{-/-}$mice were intravenously administered with ex vivo IL-4/13-produced BMDM (SIRP $\alpha^{\text {high }}$ M, 1x107, GFP ${ }^{+}$) on D6 and D9 (E); body weight change and fecal Lcn-2 were monitored (F). Data in each panel represent at least three independent experiments with $\mathrm{n} \geq 3$ if applicable. Error bars are \pm SEM. Statistical significance was determined by two-way ANOVA with Tukey's multiple comparisons test $(\mathbf{A}, \mathbf{F})$ or Student's t test $(\mathbf{B}, \mathbf{C})$, where $* \mathrm{p}<0.05, * * \mathrm{p}<0.01$ and $* * * \mathrm{p}<0.001$. 
$A$

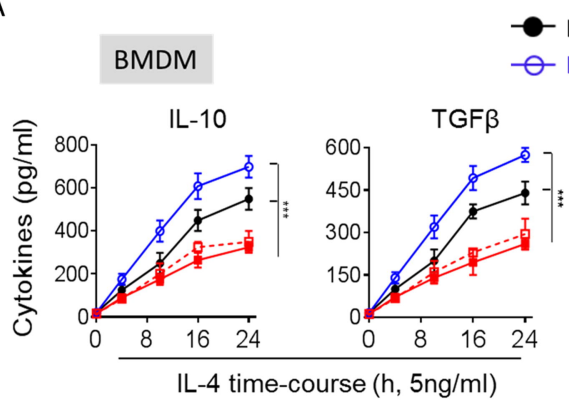

D

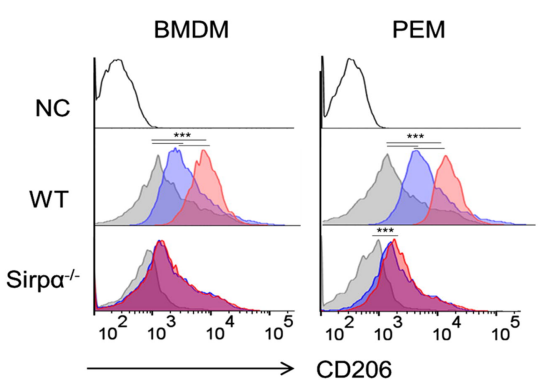

WT+mCD47.ex - $\square$ Sirpa $^{-/-m C D 47 . e x}$

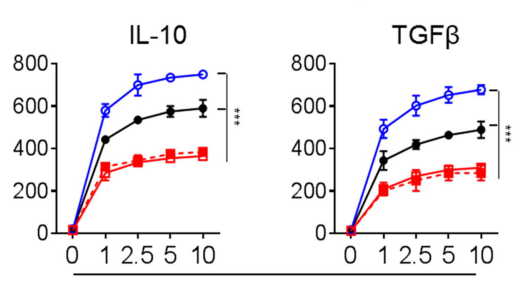

IL-4 dose (ng/ml, 16h)

E
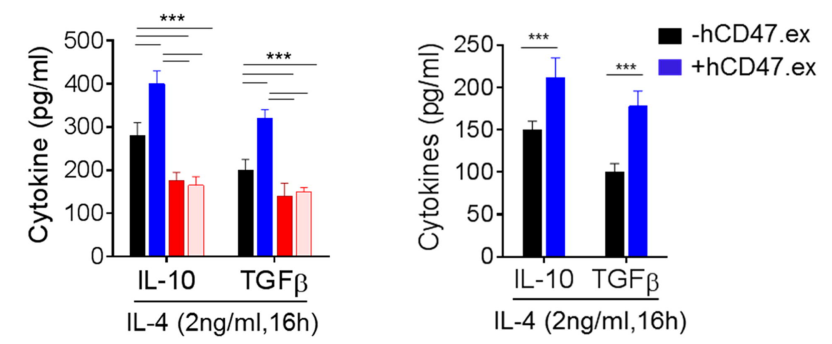

$\mathrm{F}$

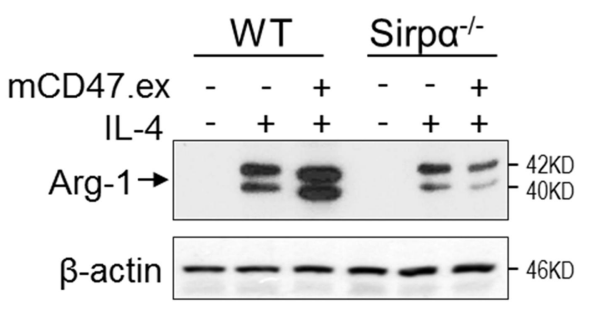

a WT

- WT+mCD47.ex

- Sirpa $^{-1-}$

$\square$ Sirpa $^{-/-}+$mCD47.ex

$\square$ No treat $\quad \square \mathrm{IL}-4 \quad \square \mathrm{IL}-4+\mathrm{mCD} 47 . e x$

G

WT $\quad$ Sirpa $^{-1-}$

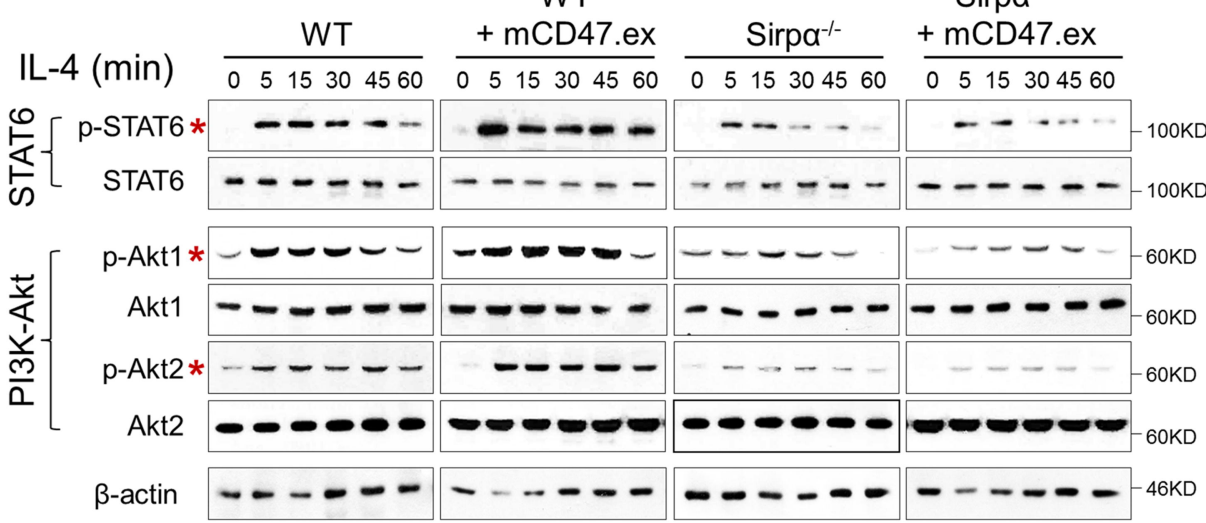

$$
\begin{aligned}
& \text { WT }- \text { WT +mCD47.ex } \\
& \text {.. Sirpa }{ }^{-/-}-\text {Sirpa }^{-/-}+\text {mCD47.ex }
\end{aligned}
$$
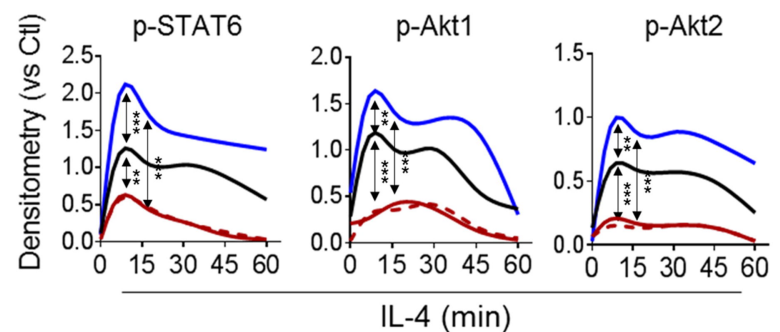

$\mathrm{H}$
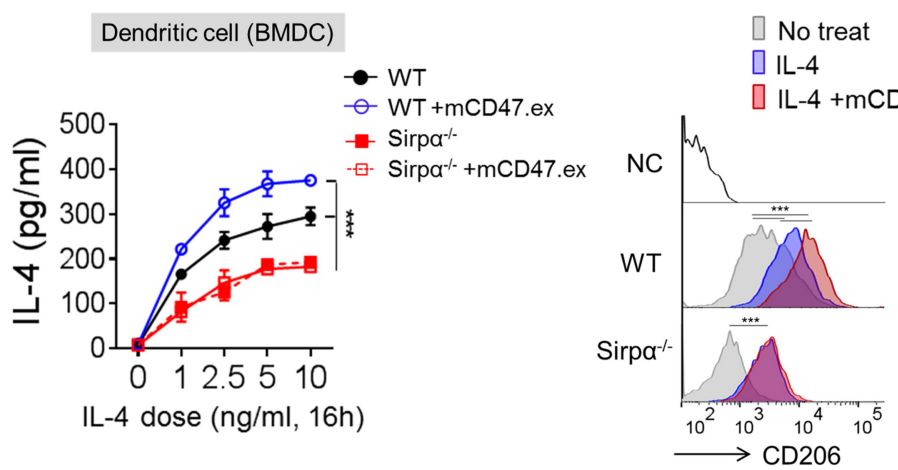

I BMDM

$\bullet W T \quad \rightarrow \mathrm{Sirpa}^{-1-}$

- WT +mCD47.ex - Sirpa $^{-1-}+$ mCD47.ex

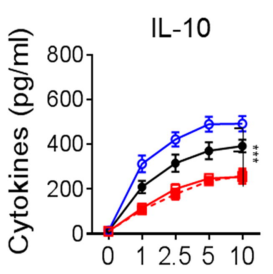

IL-13 dose (ng/ml, 16h)

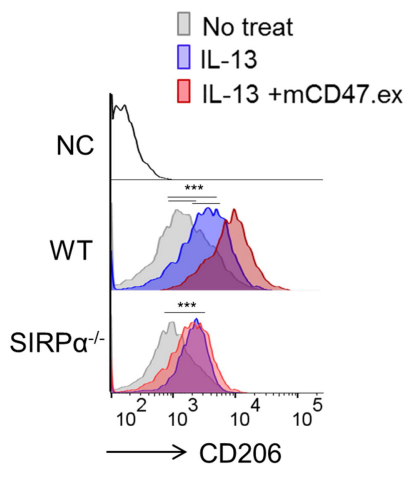

Fig. 1 

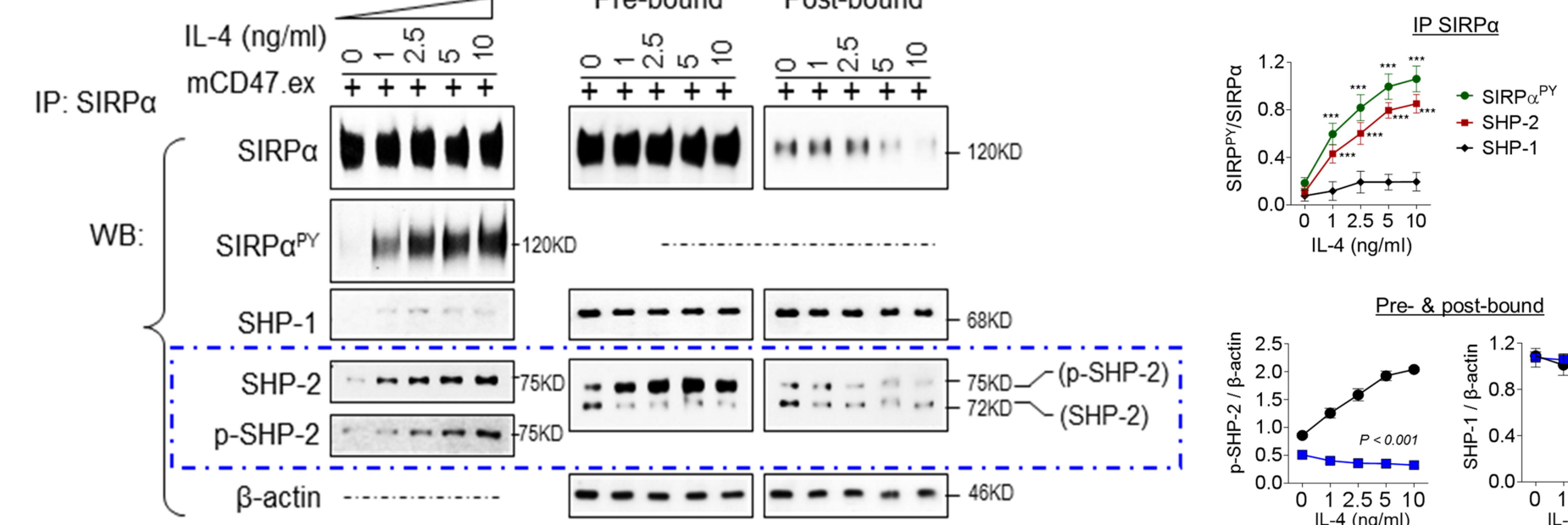

B

IL-4 (5ng/ml, min) $0 5 2 0 \longdiv { 5 2 0 }$

IP: SIRPa mCD47.ex $--\overline{+++}$
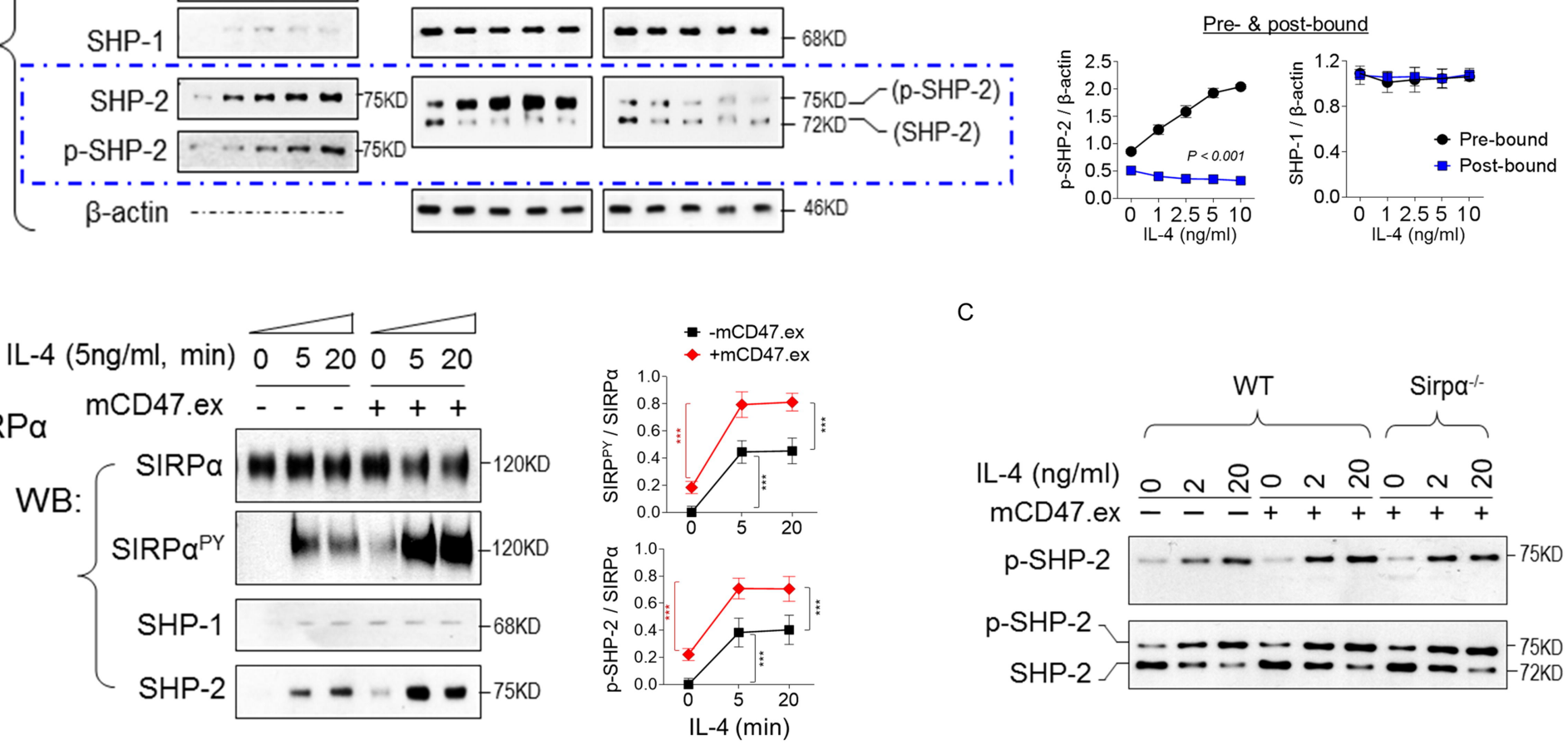

C

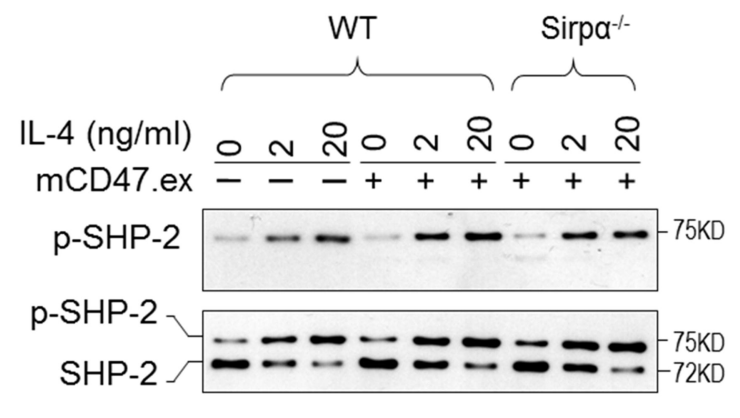

E

IP: SIRPa

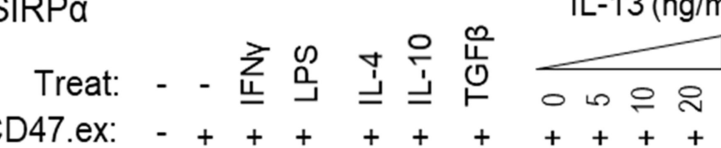
mCD47.ex:

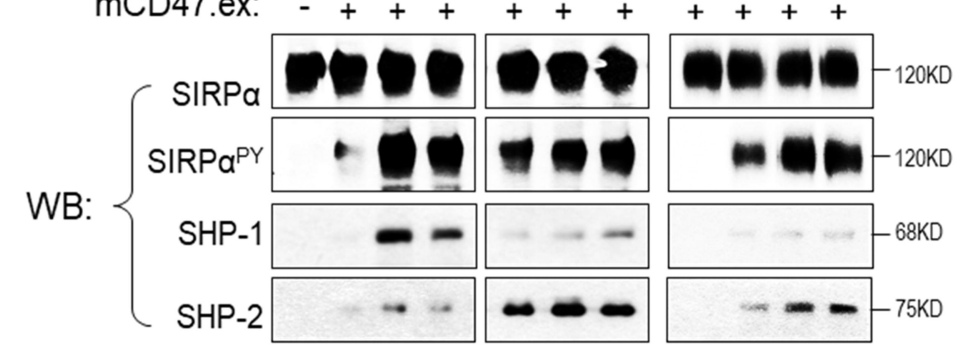

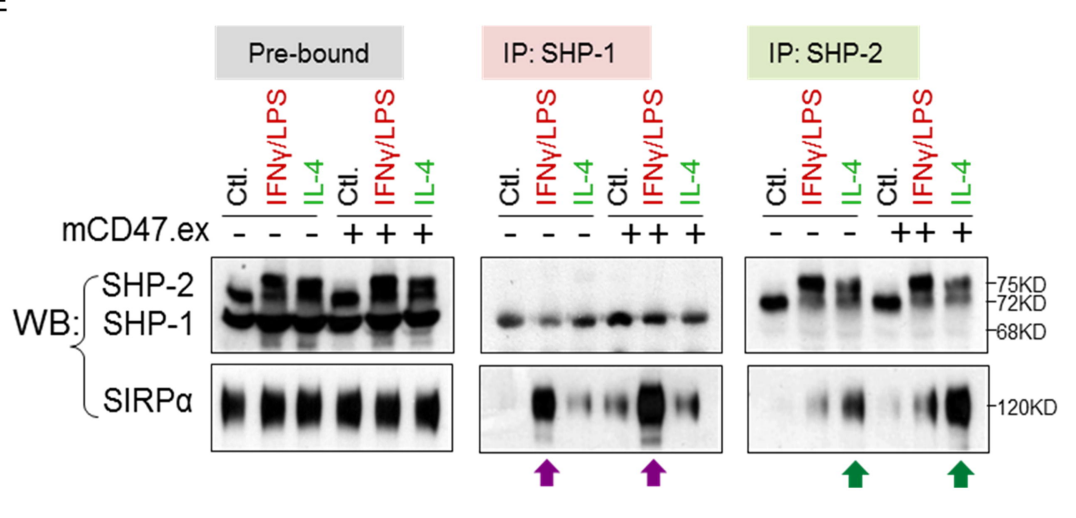

Fig.2 
A

IP: SIRPa

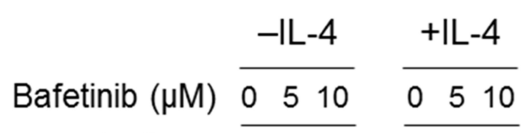

mCD47.ex

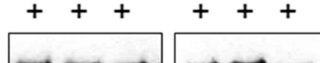

WB:

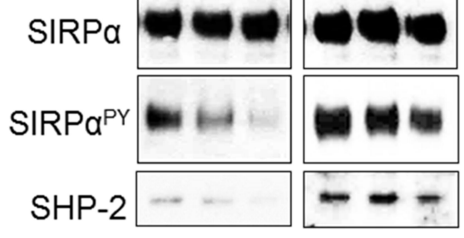

+IL-4

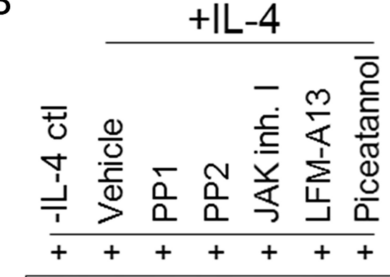

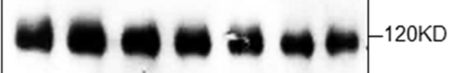

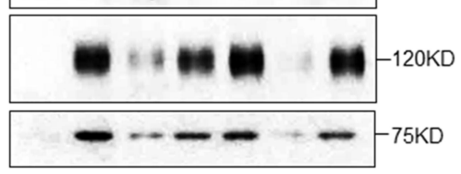

C +IL-4

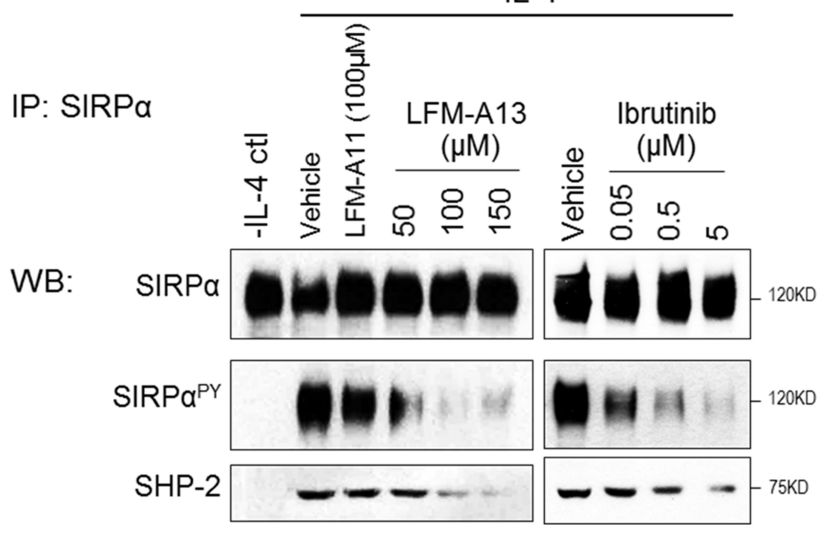

F
D

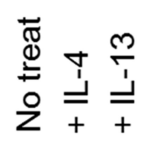

p-BTK - - -77KD

BTK - $--77 \mathrm{~T} \mathrm{D}$

$\beta$-actin ---60 -
E

$+\mathrm{IL}-4$

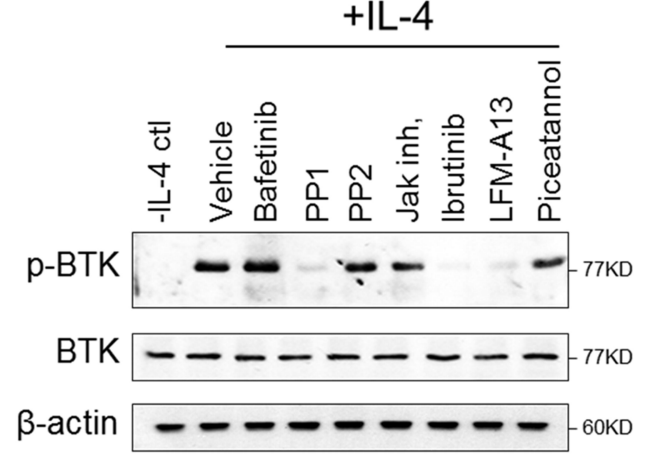

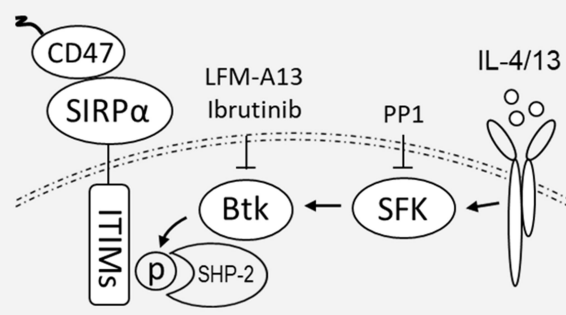




\section{IP: SHP-2}

WT Sirpa ${ }^{-/}$

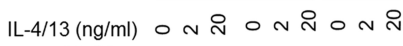
mCD47.ex

- + + +++

Receptor Complex:

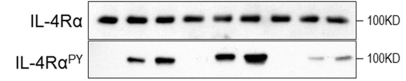

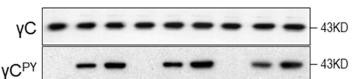

IL-13Ra1 - - - - - - - - - 55 K

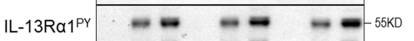

Downstream:
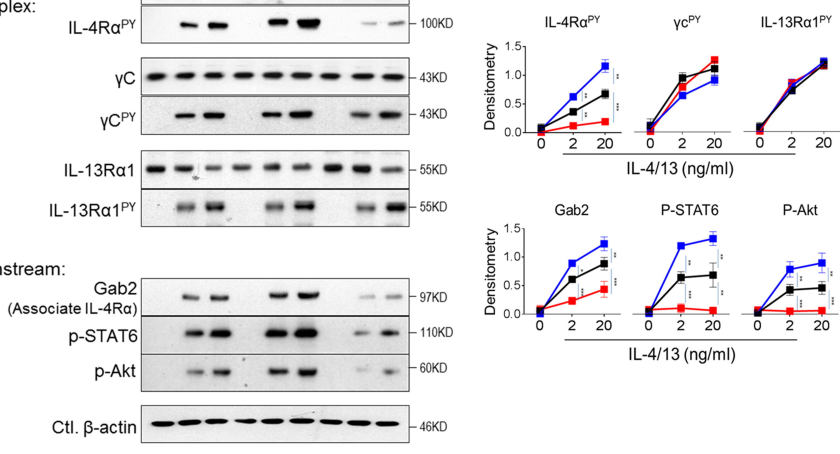

$\mathrm{E}$
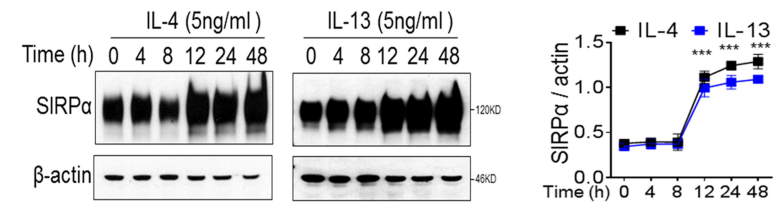

$\mathrm{F}$
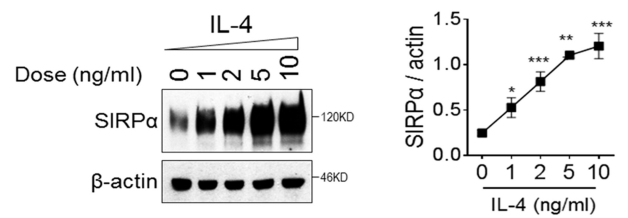

C

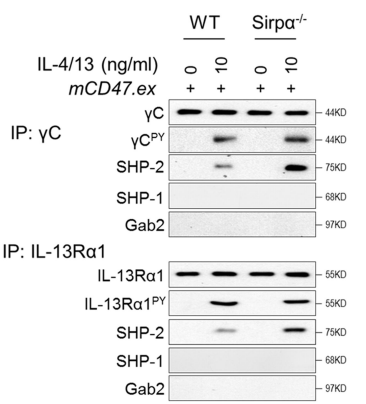

$$
\text { IP: IL-4Ra IL }
$$

$\mathrm{LL}-4 / 13(\mathrm{ng} / \mathrm{ml})$

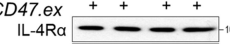

WT Sirpar

\begin{tabular}{ll}
\hline$-4 R a^{P Y}$ & -1 \\
SHP-2 & \\
SHP-1 &
\end{tabular}

SHP-1
Gab2

$\mathrm{IL}-4 \mathrm{Ra}-\mathrm{C}-\mathrm{-10000}^{-100}$

$\mathrm{IL}-4 \mathrm{Ra}^{\mathrm{PY}}-{ }_{-10000}^{-1000}$

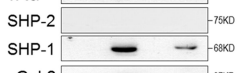

Gab2 - -

G

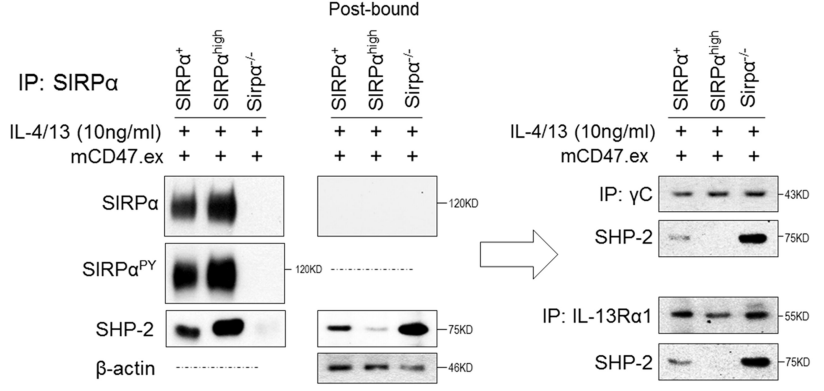

D IP: IL-4Ra
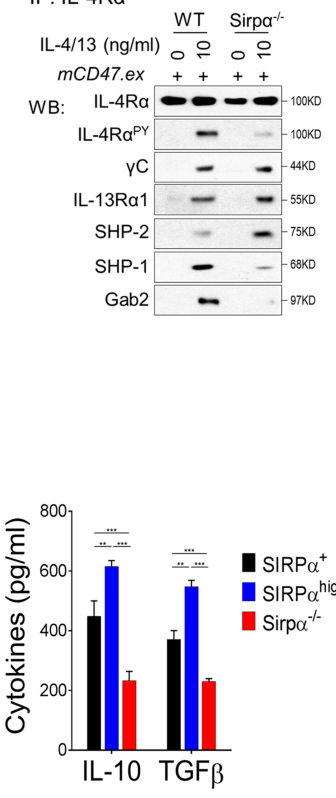
IL-4/13 (5ng/ml)
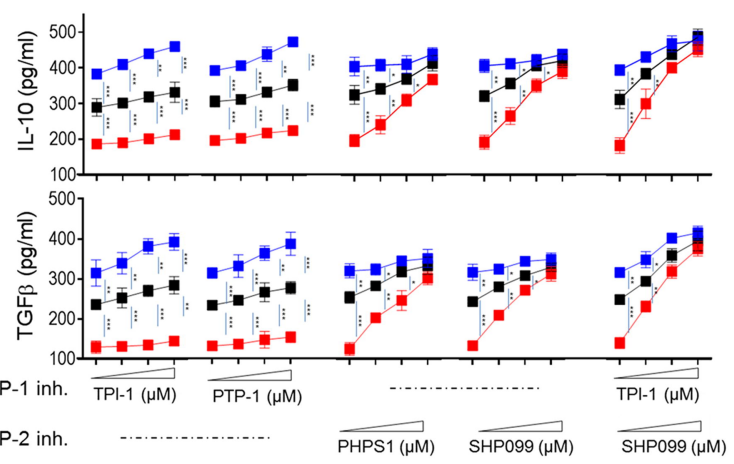

WT $\quad$ Sirpo $^{-/-}$

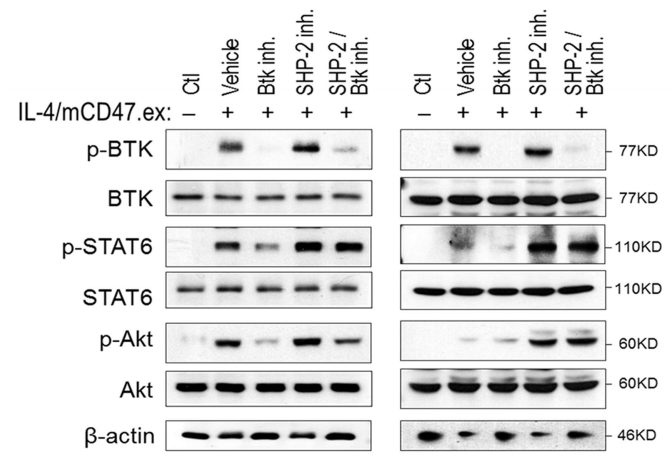

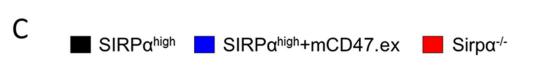
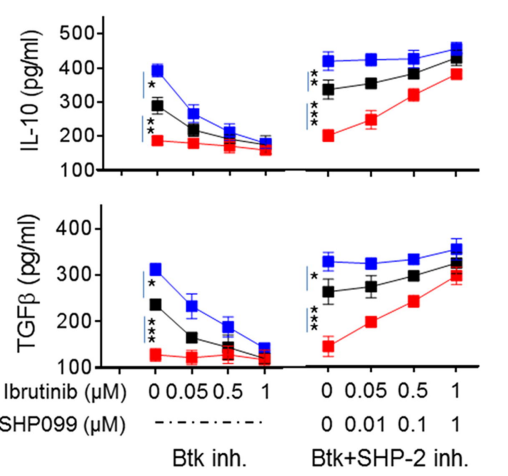

D

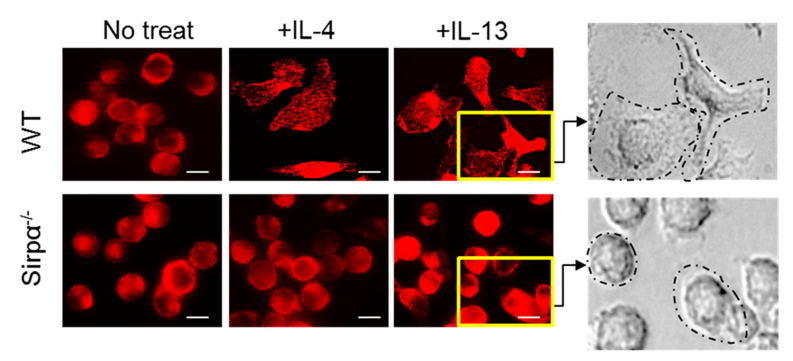

$+\mathrm{IL}-4$

Btk inh. SHP-2 inh. SHP-2 / Btk inh.

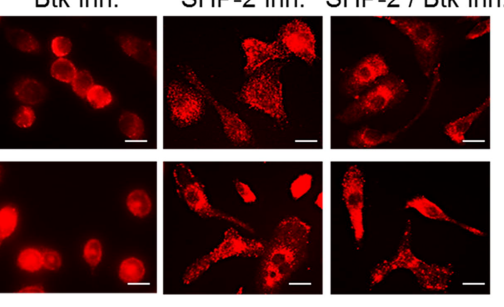

WT + IL-4

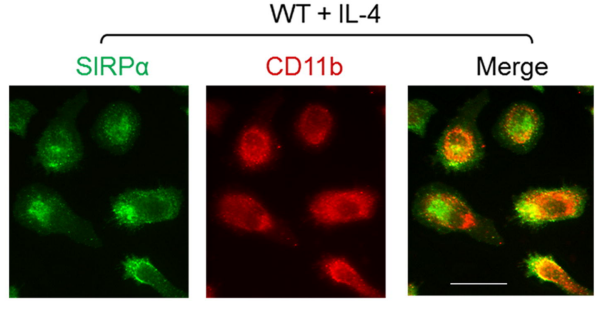

Fig. 5 

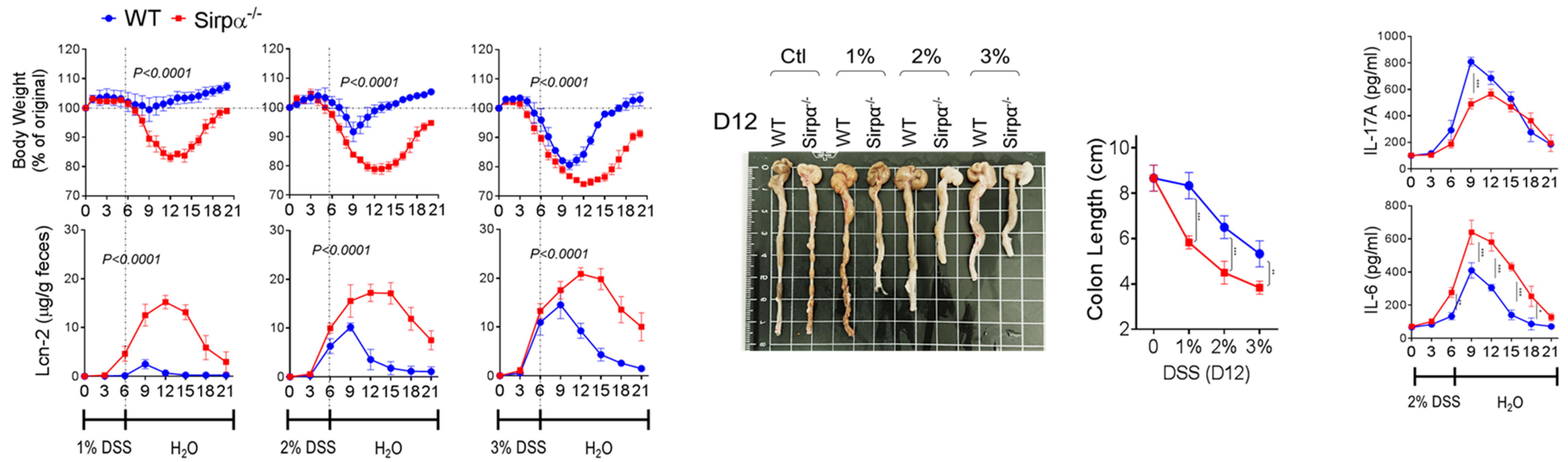

D

3\% DSS, D12

IL-10

E

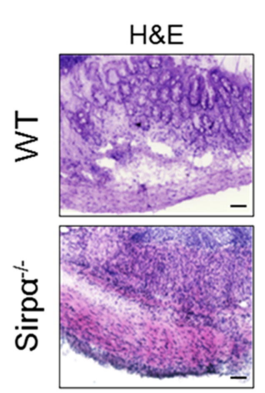

IL-10

DAPI $\quad F 4 / 80$

IL-6

I.v. IL-4-treated WT BMDM

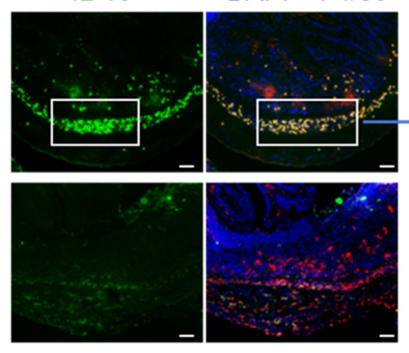

$\mathrm{IL}-10^{+}$macrophage (green) $\mathrm{F} 4 / 80^{+}$macrophage (red)
DAPI Ly6G

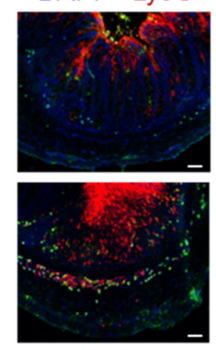

IL- $6^{+}$macrophage (green) Ly6G+ PMN (red)

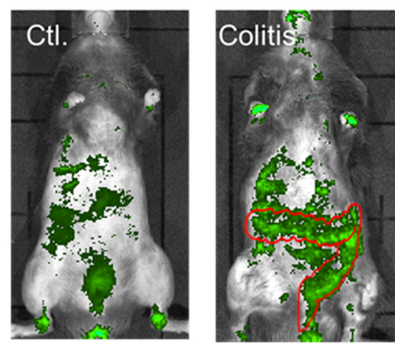

Sirpa $^{-/-}$recipient

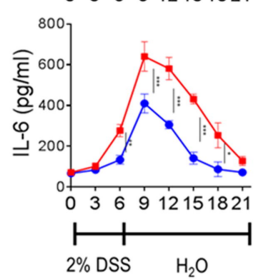

$\mathrm{F} \leftarrow \mathrm{WT}$

${ }_{120}-\mathrm{Sirp \alpha}^{-/-}+$irpo. $\alpha^{- \text {i.v. SIRPa }}{ }^{\text {high } M}$

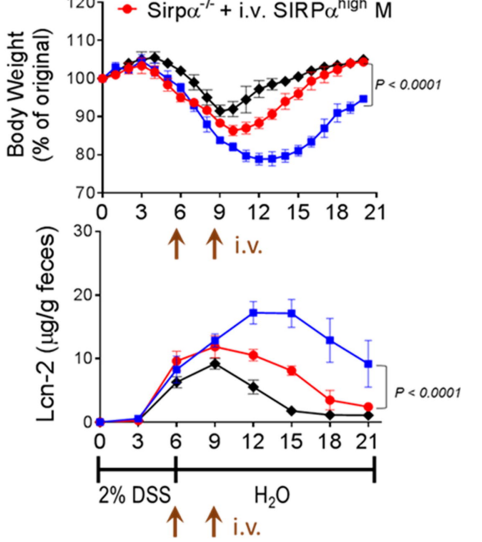

Fig. 6 